\title{
Grafting Aminocyclopentane Carboxylic Acids onto the RGD Tripeptide Sequence Generates Low Nanomolar $\alpha_{V} \beta_{3} / \alpha_{V} \beta_{5}$ Integrin Dual Binders
}

Giovanni Casiraghi, Gloria Rassu, Luciana Auzzas, Paola Burreddu, Enrico Gaetani, Lucia Battistini, Franca Zanardi, Claudio Curti, Giuseppe Nicastro, Laura Belvisi, Ilaria Motto, Massimo Castorina,

Giuseppe Giannini, and Claudio Pisano<smiles></smiles>

$\alpha_{V} \beta_{3} I C_{50}: 4.60 \mathrm{nM}$ $\alpha_{v} \beta_{5} I_{50}: 3.43 \mathrm{nM}$<smiles>N=C(N)NCCCC(NC(=O)C1CC[C@@H](NC(=O)C(CC(=O)O)NC(=O)CNCC(=O)O)C1)C(=O)O</smiles>

$\alpha_{V} \beta_{3} I C_{50}: 1.50 \mathrm{nM}$ $\alpha_{v} \beta_{5} I C_{50}: 0.59 \mathrm{nM}$

giovanni.casiraghi@unipr.it

\section{SUPPORTING INFORMATION}

- Title Page and Table of Contents

S1

- Experimental Procedures and Spectroscopic Data for Compounds

14-22 and 30-36

S2

- NMR Data of Cyclopeptides 1-4 (Table S1) S8

- NMR Data of Cyclopeptides 5-7 (Table S2) S9

- NMR Data of Cyclopeptides 8-11 (Table S3) S10

- Torsion Angles $\phi$ and $\psi$ of Compounds 1, 3, 6, 7, 9, 10 (Table S4) S11

- Copies of ${ }^{1} \mathrm{H}$ NMR Spectra $\left(600 \mathrm{MHz}, \mathrm{D}_{2} \mathrm{O}\right)$ of Cyclopeptides 1-11 $\quad \mathrm{S} 12$

- Elemental Analysis Data (Table S5) S23

$\begin{array}{lr}\text { - References } & \text { S24 }\end{array}$ 
Chemistry. Materials and Methods. All solid-phase synthesis chemicals were dried in dessicator before use; all other chemicals were used as supplied without further purification. Apart from Nmethylpyrrolidone (NMP), all organic solvents were dried and freshly distilled before use according to literature procedures. Commercial Fmoc-amino acids were purchased from Fluka or Bachem; cTrt resin (2-chlorotrityl chloride polymer bound, 100-300 mesh, nominal loading $\sim 1.9 \mathrm{mmol} / \mathrm{g}$, calculated loading 1.55-1.8 mmol/g) was from Fluka, coupling reagents were from either Bachem or Aldrich and all other chemicals and reagents were from either Aldrich or Acros. All moisture sensitive reactions were carried out under a positive pressure of nitrogen or argon. TNBS test was performed according to the following procedure. A few resin beads were sampled and accurately washed with ethanol. The sample was then placed in a vial and 1 drop of a $10 \%$ solution of DIEA in DMF and 1 drop of $1 \%$ 2,4,6-trinitrobenzenesulfonic acid (TNBS) in DMF were added. The sample was then observed under a microscope and color changes noted. The TNBS test is considered positive (presence of free amino groups) when the resin beads turn orange or red within 1 min and negative (no free amino groups) when the beads remain colorless.

TLC analysis was performed on silica gel $60 \mathrm{~F}_{254}$ plates (Merck) with visualization under shortwavelength UV light or by dipping the plates with molybdate reagent $\left(\mathrm{H}_{2} \mathrm{O} /\right.$ concentrated $\left.\mathrm{H}_{2} \mathrm{SO}_{4} /\left(\mathrm{NH}_{4}\right)_{6} \mathrm{Mo}_{7} \mathrm{O}_{24} \cdot 4 \mathrm{H}_{2} \mathrm{O} / \mathrm{Ce}_{2}\left(\mathrm{SO}_{4}\right)_{3} \quad 90 / 10 / 25 / 1 \quad \mathrm{v} / \mathrm{v} / \mathrm{w} / \mathrm{w}\right)$ followed by heating. Flash chromatography was performed on 40-63 $\mu \mathrm{m}$ silica gel from Merck using the indicated solvent mixtures. Melting points were determined with an optical thermomicroscope Optiphot2-Pol Nikon and are uncorrected. Optical rotations were measured using a Perkin-Elmer model 341 polarimeter at ambient temperature using a $100-\mathrm{mm}$ cell with a $1-\mathrm{mL}$ capacity and are given in units of $10^{-1} \mathrm{deg} \mathrm{cm}^{2}$ $\mathrm{g}^{-1}$.

${ }^{1} \mathrm{H}$ and ${ }^{13} \mathrm{C}$ NMR spectra were recorded on a Bruker Avance 300 operating at $300 / 75 \mathrm{MHz}$, or a Varian Mercury Plus MP-400 at 400/100 MHz, or a Varian Inova SB-600 at 600/150 MHz, or a Varian Inova 800 at $800 / 200 \mathrm{MHz}$.

Analytical reversed-phase HPLC was performed on a SpectraSystem P2000 apparatus from Thermo Separation Products equipped with a Discovery C18-10 $\mu \mathrm{m}$ column $(250 \times 4.6 \mathrm{~mm})$. Semipreparative reversed-phase HPLC was performed on the same apparatus equipped with a Discovery C18-10 $\mu \mathrm{m}$ column $(250 \times 10 \mathrm{~mm})$. Direct infusion ESI-MS spectra were recorded on Applied Biosystems API 150EX apparatus. High-resolution mass spectrometry (HRMS) measurements were performed on a Bruker APEX IIIQ Fourier transform mass spectrometer equipped with an external electrospray ion source. Elemental analyses were performed by the Microanalytical Laboratory of the University of Parma.

$N$-(tert-Butoxycarbonyl)-2-[(tert-butyldimethylsilyl)oxy]pyrrole (12) was prepared from pyrrole according to a described protocol. ${ }^{1}$ 2,3-O-Isopropylidene-D-glyceraldehyde $(R-13)$ was prepared from D-mannitol according to a recently optimized protocol. ${ }^{2}$ The preparation of $2,3-O$-isopropylidene-Lglyceraldehyde $(S-13)$ was carried out starting from 5,6-O-isopropylidene-L-gulonic acid 1,4-lactone following a known protocol. ${ }^{3}$ Amino acids 23-29 were purchased from Acros and used without further purification.

(1'S,4" $R, 5 R)-1-($ tert-Butoxycarbonyl)-5-[(2,2-dimethyl-[1,3]dioxolan-4-yl)-hydroxymethyl]-1,5dihydropyrrol-2-one (14). To a stirring solution of silyloxypyrrole 12 (1.73 g, $5.83 \mathrm{mmol})$ in anhydrous $\mathrm{Et}_{2} \mathrm{O}(50 \mathrm{~mL})$ under argon atmosphere at $-90{ }^{\circ} \mathrm{C}$ was added an ether solution of aldehyde $R$ $13\left(0.91 \mathrm{~g}, 7.0 \mathrm{mmol}\right.$ dissolved in $20 \mathrm{~mL}$ of $\left.\mathrm{Et}_{2} \mathrm{O}\right)$. The resulting mixture was allowed to cool to $-90{ }^{\circ} \mathrm{C}$ for $10 \mathrm{~min}$, then $\mathrm{SnCl}_{4}\left(7.58 \mathrm{~mL}\right.$ of a $1.0 \mathrm{M}$ solution in $\left.\mathrm{CH}_{2} \mathrm{Cl}_{2}, 7.58 \mathrm{mmol}\right)$ was slowly added dropwise to the mixture at the same temperature over a period of $30 \mathrm{~min}$. After $3 \mathrm{~h}$, the reaction mixture was quenched at $-90{ }^{\circ} \mathrm{C}$ with saturated aqueous $\mathrm{NaHCO}_{3}(20 \mathrm{~mL})$ and solid $\mathrm{NaHCO}_{3}(1.3 \mathrm{~g})$ and temperature was allowed to reach ambient value $\left(20^{\circ} \mathrm{C}\right)$. Further portions of solid $\mathrm{NaHCO}_{3}$ were added 
until neutral $\mathrm{pH}$ was achieved. The mixture was concentrated under vacuum to remove the ethereal phase. The water mixture was washed with hexanes $(2 \times 10 \mathrm{~mL})$, and then extracted with EtOAc $(3 \times$ $10 \mathrm{~mL})$. The combined organic layers were dried $\left(\mathrm{MgSO}_{4}\right)$ and concentrated in vacuum to give a solid crude residue which was crystallized from EtOAc/hexane to give unsaturated lactam $14(1.50 \mathrm{~g}, 82 \%$, de $\geq 98 \%)$ as a white solid: $\operatorname{mp~} 138-140^{\circ} \mathrm{C} ;[\alpha]_{\mathrm{D}}{ }^{20}+197.6\left(c 0.8, \mathrm{CHCl}_{3}\right) ;{ }^{1} \mathrm{H} \mathrm{NMR}\left(300 \mathrm{MHz}, \mathrm{CDCl}_{3}\right)$ $\delta 7.43(\mathrm{dd}, J=6.3,2.1 \mathrm{~Hz}, 1 \mathrm{H}), 6.13(\mathrm{dd}, J=6.3,1.5 \mathrm{~Hz}, 1 \mathrm{H}), 4.81(\mathrm{dt}, J=5.7,2.4 \mathrm{~Hz}, 1 \mathrm{H}), 4.09$ (ddd, $J=6.0,5.7,3.9 \mathrm{~Hz}, 1 \mathrm{H}), 4.01(\mathrm{q}, J=6.0 \mathrm{~Hz}, 1 \mathrm{H}), 3.94(\mathrm{dd}, J=8.1,6.0 \mathrm{~Hz}, 1 \mathrm{H}), 3.86(\mathrm{dd}, J=8.1,6.0$ $\mathrm{Hz}, 1 \mathrm{H}), 3.63(\mathrm{~d}, J=3.9 \mathrm{~Hz}, 1 \mathrm{H}), 1.57(\mathrm{~s}, 9 \mathrm{H}), 1.37(\mathrm{~s}, 3 \mathrm{H}), 1.32(\mathrm{~s}, 3 \mathrm{H}) ;{ }^{13} \mathrm{C}$ NMR $\left(75 \mathrm{MHz}, \mathrm{CDCl}_{3}\right)$ $\delta$ 168.9, 150.9, 148.2, 126.9, 109.2, 83.8, 75.6, 72.6, 66.4, 65.6, 28.0 (3C), 26.4, 25.1. Anal. $\left(\mathrm{C}_{15} \mathrm{H}_{23} \mathrm{NO}_{6}\right): \mathrm{C}, \mathrm{H}, \mathrm{N}$.

$(1 ' S, 4 " R, 5 R)-5-[($ tert-Butyldimethylsilanyloxy)-(2,2-dimethyl-[1,3]dioxolan-4-

yl)methyl]pyrrolidin-2-one (15). Palladium on carbon $(10 \%, 160 \mathrm{mg})$ was added to a stirring solution of unsaturated lactam $14(1.50 \mathrm{~g}, 4.78 \mathrm{mmol})$ in anhydrous EtOAc $(25 \mathrm{~mL})$ in the presence of a small amount of $\mathrm{NaOAc}(66 \mathrm{mg})$ at room temperature. The reaction vessel was evacuated by aspirator and thoroughly purged with hydrogen (three times), and the resulting heterogeneous mixture was stirred under a hydrogen balloon. After $24 \mathrm{~h}$, the hydrogen was evacuated, the catalyst filtered off, and the filtrate concentrated under vacuum to give a crude residue that was crystallized from EtOAc/hexane $(1: 3)$ to give a saturated lactam intermediate $(1.48 \mathrm{~g}, 98 \%)$ as a white solid: $\mathrm{mp} 102-105^{\circ} \mathrm{C} ;[\alpha]_{\mathrm{D}}{ }^{20}$ $-60.1\left(c 1.0, \mathrm{CHCl}_{3}\right) ;{ }^{1} \mathrm{H} \mathrm{NMR}\left(300 \mathrm{MHz}, \mathrm{CDCl}_{3}\right) \delta 4.31(\mathrm{ddd}, J=5.7,5.4,3.9 \mathrm{~Hz}, 1 \mathrm{H}), 4.05(\mathrm{~m}, 2 \mathrm{H})$, 3.97 (ddd, $J=5.5,4.8,1.2 \mathrm{~Hz}, 1 \mathrm{H}), 3.69(\mathrm{q}, J=5.7 \mathrm{~Hz}, 1 \mathrm{H}), 3.54(\mathrm{~d}, J=6.3 \mathrm{~Hz}, 1 \mathrm{H}), 2.71(\mathrm{dt}, J=$ $17.1,10.5 \mathrm{~Hz}, 1 \mathrm{H}), 2.32$ (ddd, $J=17.7,6.0,4.8 \mathrm{~Hz}, 1 \mathrm{H}), 2.10(\mathrm{~m}, 2 \mathrm{H}), 1.48(\mathrm{~s}, 9 \mathrm{H}), 1.36(\mathrm{~s}, 3 \mathrm{H}), 1.30$ (s, 3H).

This intermediate $(1.48 \mathrm{~g}, 4.68 \mathrm{mmol})$ was dissolved in anhydrous $\mathrm{CH}_{2} \mathrm{Cl}_{2}(20 \mathrm{~mL})$ at ambient temperature under argon and 2,6-lutidine $(1.65 \mathrm{~mL}, 15.44 \mathrm{mmol})$ and tert-butyldimethylsilyl trifluoromethanesulfonate (TBSOTf, $1.18 \mathrm{~mL}, 5.15 \mathrm{mmol}$ ) were sequentially added under stirring. After $3 \mathrm{~h}$, the reaction mixture was quenched with 5\% aqueous citric acid, diluted with water and extracted with $\mathrm{CH}_{2} \mathrm{Cl}_{2}(3 \times 10 \mathrm{~mL})$. The combined organic layers were dried $\left(\mathrm{MgSO}_{4}\right)$, filtered, and concentrated under reduced pressure to furnish a crude product which was purified by flash chromatography (EtOAc/hexane 6:4). A protected lactam intermediate was obtained $(1.91 \mathrm{~g}, 95 \%)$ as a pale yellow oil: $[\alpha]_{\mathrm{D}}{ }^{20}+38.1\left(c 0.8, \mathrm{CHCl}_{3}\right) ;{ }^{1} \mathrm{H} \mathrm{NMR}\left(300 \mathrm{MHz}, \mathrm{CDCl}_{3}\right) \delta 4.19$ (ddd, $J=9.2,3.7$, $1.3 \mathrm{~Hz}, 1 \mathrm{H}), 4.10(\mathrm{dd}, J=8.5,3.7 \mathrm{~Hz}, 1 \mathrm{H}), 4.07(\mathrm{dd}, J=8.0,5.9 \mathrm{~Hz}, 1 \mathrm{H}), 3.95(\mathrm{dt}, J=8.5,6.1 \mathrm{~Hz}$, $1 \mathrm{H}), 3.71(\mathrm{dd}, J=8.1,6.2 \mathrm{~Hz}, 1 \mathrm{H}), 2.57$ (ddd, $J=17.8,11.5,9.8 \mathrm{~Hz}, 1 \mathrm{H}), 2.38$ (ddd, $J=17.8,10.3,2.0$ $\mathrm{Hz}, 1 \mathrm{H}), 2.16(\mathrm{~m}, 1 \mathrm{H}), 1.98(\mathrm{~m}, 1 \mathrm{H}), 1.52(\mathrm{~s}, 9 \mathrm{H}), 1.30(\mathrm{~s}, 3 \mathrm{H}), 1.26(\mathrm{~s}, 3 \mathrm{H}), 0.85(\mathrm{~s}, 9 \mathrm{H}), 0.12(\mathrm{~s}, 3 \mathrm{H})$, $0.10(\mathrm{~s}, 3 \mathrm{H}) ;{ }^{13} \mathrm{C}$ NMR $\left(75 \mathrm{MHz}, \mathrm{CDCl}_{3}\right) \delta 174.4,149.8,109.8,82.3,75.2,71.1,68.6,60.2,31.8,29.4$, 28.0 (3C), 26.2, 25.4 (3C), 24.9, 17.6, -4.2, -5.0 .

To a solution of this intermediate $(1.91 \mathrm{~g}, 4.45 \mathrm{mmol})$ in $60 \mathrm{~mL}$ of acetonitrile, warmed to $80{ }^{\circ} \mathrm{C}$, ammonium cerium (IV) nitrate (CAN, $515 \mathrm{mg}, 0.94 \mathrm{mmol}$ ) was added portionwise. After $4 \mathrm{~h}$, the reaction was quenched with $\mathrm{NaHCO}_{3}$ (aq, sat.) and extracted with EtOAc $(3 \times 50 \mathrm{~mL})$. The combined organic layers were dried $\left(\mathrm{MgSO}_{4}\right)$, filtered and concentrated under reduced pressure furnishing a crude residue which was purified by flash chromatography (EtOAc). $N$-deprotected lactam 15 (1.36 g, 93\%) was recovered as a colorless oil: $[\alpha]_{\mathrm{D}}{ }^{20}-6.8\left(c 10.9, \mathrm{CHCl}_{3}\right) ;{ }^{1} \mathrm{H} \mathrm{NMR}\left(300 \mathrm{MHz}, \mathrm{CDCl}_{3}\right) \delta 5.80$ (bs, $1 \mathrm{H}), 4.00(\mathrm{~m}, 2 \mathrm{H}), 3.76(\mathrm{~m}, 1 \mathrm{H}), 3.67(\mathrm{~m}, 1 \mathrm{H}), 3.60(\mathrm{~m}, 1 \mathrm{H}), 2.29(\mathrm{~m}, 2 \mathrm{H}), 2.17(\mathrm{~m}, 1 \mathrm{H}), 1.91(\mathrm{~m}$, $1 \mathrm{H}), 1.38(\mathrm{~s}, 3 \mathrm{H}), 1.31(\mathrm{~s}, 3 \mathrm{H}), 0.88(\mathrm{~s}, 9 \mathrm{H}), 0.09(\mathrm{~s}, 3 \mathrm{H}), 0.07(\mathrm{~s}, 3 \mathrm{H}) ;{ }^{13} \mathrm{C} \mathrm{NMR}\left(75 \mathrm{MHz}, \mathrm{CDCl}_{3}\right) \delta$ $177.7,108.9,76.7,74.8,66.0,56.2,29.7,26.2,25.6$ (3C), 24.8, 23.3, 17.8, -4.3, -4.4. Anal. $\left(\mathrm{C}_{16} \mathrm{H}_{31} \mathrm{NO}_{4} \mathrm{Si}\right): \mathrm{C}, \mathrm{H}, \mathrm{N}$.

(2S,2'R)-(1-Benzyl-5-oxopyrrolidin-2-yl)-(tert-butyldimethylsilanyloxy)acetaldehyde

(16).

Lactam 15 (1.36 g, $4.14 \mathrm{mmol})$ and $\mathrm{NaH}(248 \mathrm{mg}, 6.21 \mathrm{mmol}, 60 \% \mathrm{wt}$. dispersion in mineral oil) were dissolved in freshly distilled THF $(100 \mathrm{~mL})$ under argon atmosphere at room temperature. The 
resulting solution was allowed to stir for $10 \mathrm{~min}$ before the addition of benzyl bromide $(\mathrm{BnBr}, 0.74 \mathrm{~mL}$, $6.21 \mathrm{mmol}$ ). The reaction mixture was refluxed for $1.5 \mathrm{~h}$, cooled to room temperature, and quenched by the addition of $5 \%$ aqueous citric acid until neutralization. The mixture was diluted with water, extracted with $\mathrm{CH}_{2} \mathrm{Cl}_{2}$, dried, and concentrated to a yellow oil. The crude product was purified by flash chromatography (EtOAc/hexane 7:3) to yield a $N$-benzyl intermediate $(1.58 \mathrm{~g}, 91 \%)$ as a colorless oil: $[\alpha]_{\mathrm{D}}{ }^{20}-13.2\left(c 1.2, \mathrm{CHCl}_{3}\right) ;{ }^{1} \mathrm{H}$ NMR $\left(300 \mathrm{MHz}, \mathrm{CDCl}_{3}\right) \delta 7.34(\mathrm{~m}, 5 \mathrm{H}), 5.23(1 / 2 \mathrm{AB} \mathrm{q}, J=15.1 \mathrm{~Hz}$, $1 \mathrm{H}), 4.06(\mathrm{~m}, 2 \mathrm{H}), 3.94(\mathrm{dd}, J=6.4,3.9 \mathrm{~Hz}, 1 \mathrm{H}), 3.92(1 / 2 \mathrm{AB} \mathrm{q}, J=14.8 \mathrm{~Hz}, 1 \mathrm{H}), 3.82(\mathrm{~m}, 1 \mathrm{H}), 3.54$ $(\mathrm{dt}, J=7.7,3.9 \mathrm{~Hz}, 1 \mathrm{H}), 2.3-2.6(\mathrm{~m}, 2 \mathrm{H}), 1.9-2.1(\mathrm{~m}, 2 \mathrm{H}), 1.47(\mathrm{~s}, 3 \mathrm{H}), 1.36(\mathrm{~s}, 3 \mathrm{H}), 0.84(\mathrm{~s}, 9 \mathrm{H}), 0.01$ $(\mathrm{s}, 3 \mathrm{H}), 0.02(\mathrm{~s}, 3 \mathrm{H}) ;{ }^{13} \mathrm{C} \mathrm{NMR}\left(75 \mathrm{MHz}, \mathrm{CDCl}_{3}\right) \delta 175.3,136.2,128.7$ (2C), $128.3(2 \mathrm{C}), 127.6,109.3$, 74.9, 71.2, 67.7, 59.1, 45.1, 30.2, 26.4, $25.6(3 \mathrm{C}), 25.0,18.5,17.8,-4.8,-4.7$.

This protected lactam intermediate $(1.58 \mathrm{~g}, 3.77 \mathrm{mmol})$ was dissolved in EtOAc $(50 \mathrm{~mL})$ and stirred while $\mathrm{H}_{5} \mathrm{IO}_{6}(1.72 \mathrm{~g}, 7.54 \mathrm{mmol})$ was added. The reaction was stirred at room temperature for $1 \mathrm{~h}$ before it was quenched by the addition of $\mathrm{Na}_{2} \mathrm{~S}_{2} \mathrm{O}_{3}(1.19 \mathrm{~g}, 7.54 \mathrm{mmol})$ and hexane $(50 \mathrm{~mL})$. The heterogeneous mixture was filtered and the filtrates were concentrated to a yellow oil. The crude product was purified by flash chromatography (EtOAc/hexane 1:1) to give aldehyde $16(1.28 . \mathrm{g}, 98 \%)$ as a colorless oil: $[\alpha]_{\mathrm{D}}{ }^{20}-63.7\left(c 6.1, \mathrm{CHCl}_{3}\right) ;{ }^{1} \mathrm{H} \mathrm{NMR}\left(300 \mathrm{MHz}, \mathrm{CDCl}_{3}\right) \delta 9.49(\mathrm{~d}, J=1.5 \mathrm{~Hz}, 1 \mathrm{H})$, $7.30(\mathrm{~m}, 3 \mathrm{H}), 7.17(\mathrm{~m}, 2 \mathrm{H}), 4.89(1 / 2 \mathrm{AB} \mathrm{q}, J=15.6 \mathrm{~Hz}, 1 \mathrm{H}), 3.99(\mathrm{dd}, J=3.2,1.5 \mathrm{~Hz}, 1 \mathrm{H}), 3.94(1 / 2$ AB q, $J=15.6 \mathrm{~Hz}, 1 \mathrm{H}), 3.79(\mathrm{dt}, J=8.7,3.0 \mathrm{~Hz}, 1 \mathrm{H}), 2.51(\mathrm{dt}, J=17.1,9.3 \mathrm{~Hz}, 1 \mathrm{H}), 2.39(\mathrm{ddd}, J=$ 17.1, 9.6, 3.6 Hz, 1H), $2.17(\mathrm{dq}, J=13.2,9.3 \mathrm{~Hz}, 1 \mathrm{H}), 1.95(\mathrm{ddt}, J=13.5,9.9,3.6 \mathrm{~Hz}, 1 \mathrm{H}), 0.92(\mathrm{~s}$, 9H), $0.05(\mathrm{~s}, 3 \mathrm{H}), 0.03(\mathrm{~s}, 3 \mathrm{H}) ;{ }^{13} \mathrm{C} \mathrm{NMR}\left(75 \mathrm{MHz}, \mathrm{CDCl}_{3}\right) \delta 202.6,175.5,135.7,128.6(2 \mathrm{C}), 127.9$ (2C), 127.5, 78.7, 58.9, 45.5, 29.7, 25.6 (3C), 21.6, 17.9, -4.7, -5.3. Anal. $\left(\mathrm{C}_{19} \mathrm{H}_{29} \mathrm{NO}_{3} \mathrm{Si}\right): \mathrm{C}, \mathrm{H}, \mathrm{N}$.

$(1 R, 4 S, 5 S, 6 S)$-2-Benzyl-5,6-bis-(tert-butyldimethylsilanyloxy)-2-azabicyclo[2.2.1]-heptan-3-one (17) and $(1 R, 4 S, 5 R, 6 S)$-2-Benzyl-5,6-bis-(tert-butyldimethylsilanyloxy)-2-azabicyclo[2.2.1]heptan3-one (18). Diisopropylethyl amine (DIEA, $1.93 \mathrm{~mL}, 11.07 \mathrm{mmol}$ ) was dissolved in anhydrous $\mathrm{CH}_{2} \mathrm{Cl}_{2}$ $(40 \mathrm{~mL})$ at room temperature under argon atmosphere and TBSOTf $(2.54 \mathrm{~mL}, 11.07 \mathrm{mmol})$ was added. The resulting solution was stirred at the same temperature for $10 \mathrm{~min}$, then aldehyde 16 (1.28 g, 3.69 mmol) dissolved in anhydrous $\mathrm{CH}_{2} \mathrm{Cl}_{2}(20 \mathrm{~mL})$ was added dropwise. After $2 \mathrm{~h}$, the reaction mixture was quenched with $\mathrm{NH}_{4} \mathrm{Cl}$ (aq, sat.), and extracted with $\mathrm{CH}_{2} \mathrm{Cl}_{2}(3 \times 30 \mathrm{~mL})$. The combined organic layers were dried $\left(\mathrm{MgSO}_{4}\right)$, filtered, and concentrated under reduced pressure. The oily residue was purified by flash chromatography (hexanes/EtOAc 85:15) to give $17(1.33 \mathrm{~g}, 78 \%)$ and $18(0.34 \mathrm{~g}$, $20 \%)$.

Compound 17: a white solid; mp 59-60 ${ }^{\circ} \mathrm{C} ;[\alpha]_{\mathrm{D}}{ }^{20}+56.7$ (c 2.1, $\left.\mathrm{CHCl}_{3}\right) ;{ }^{1} \mathrm{H}$ NMR $(300 \mathrm{MHz}$, benzene- $\left.d_{6}\right) \delta 7.1-7.3(\mathrm{~m}, 5 \mathrm{H}), 4.64(1 / 2 \mathrm{AB} \mathrm{q}, J=14.7 \mathrm{~Hz}, 1 \mathrm{H}), 4.02(\mathrm{dt}, J=4.3,1.2 \mathrm{~Hz}, 1 \mathrm{H}), 3.97$ $(1 / 2 \mathrm{AB} \mathrm{q}, J=14.7 \mathrm{~Hz}, 1 \mathrm{H}), 3.51(\mathrm{dt}, J=2.3,1.2 \mathrm{~Hz}, 1 \mathrm{H}), 3.03(\mathrm{~m}, 1 \mathrm{H}), 2.64(\mathrm{dq}, J=4.2,1.6 \mathrm{~Hz}, 1 \mathrm{H})$, $1.71(\mathrm{dt}, J=9.9,1.6 \mathrm{~Hz}, 1 \mathrm{H}), 1.61(\mathrm{dq}, J=9.8,1.9 \mathrm{~Hz}, 1 \mathrm{H}), 1.11(\mathrm{~s}, 9 \mathrm{H}), 0.91(\mathrm{~s}, 9 \mathrm{H}), 0.32(\mathrm{~s}, 3 \mathrm{H})$, $0.15(\mathrm{~s}, 3 \mathrm{H}),-0.05(\mathrm{~s}, 3 \mathrm{H}),-0.11(\mathrm{~s}, 3 \mathrm{H}) ;{ }^{13} \mathrm{C}$ NMR $\left(75 \mathrm{MHz}\right.$, benzene- $\left.d_{6}\right) \delta 173.0,138.6,129.0(2 \mathrm{C})$, 128.7 (2C), 127.2, 82.0, 80.4, 63.1, 51.0, 45.3, 36.3, 26.0 (3C), 25.8 (3C), 18.2, 18.0, -4.1, -4.8 (2C), -5.0. Anal. $\left(\mathrm{C}_{25} \mathrm{H}_{43} \mathrm{NO}_{3} \mathrm{Si}_{2}\right): \mathrm{C}, \mathrm{H}, \mathrm{N}$.

Compound 18: a colorless oil; $[\alpha]_{\mathrm{D}}{ }^{20}-2.0\left(c 2.0, \mathrm{CHCl}_{3}\right) ;{ }^{1} \mathrm{H}$ NMR $\left(300 \mathrm{MHz}\right.$, benzene- $\left.d_{6}\right) \delta 7.1-$ $7.3(\mathrm{~m}, 5 \mathrm{H}), 4.32(1 / 2 \mathrm{AB} \mathrm{q}, J=14.8 \mathrm{~Hz}, 1 \mathrm{H}), 4.06(\mathrm{dd}, J=5.7,1.6 \mathrm{~Hz}, 1 \mathrm{H}), 3.98(1 / 2 \mathrm{AB} \mathrm{q}, J=14.8$ $\mathrm{Hz}, 1 \mathrm{H}), 3.55(\mathrm{dd}, J=5.7,1.8 \mathrm{~Hz}, 1 \mathrm{H}), 3.11(\mathrm{q}, J=2.1 \mathrm{~Hz}, 1 \mathrm{H}), 2.67(\mathrm{q}, J=1.7 \mathrm{~Hz}, 1 \mathrm{H}), 2.12(\mathrm{dt}, J=$ 9.5, 1.5 Hz, 1H), 1.53 (dquint, $J=9.5,1.7 \mathrm{~Hz}, 1 \mathrm{H}), 0.92(\mathrm{~s}, 9 \mathrm{H}), 0.85(\mathrm{~s}, 9 \mathrm{H}), 0.08(\mathrm{~s}, 3 \mathrm{H}), 0.04(\mathrm{~s}$, $3 \mathrm{H}),-0.04(\mathrm{~s}, 3 \mathrm{H}),-0.13(\mathrm{~s}, 3 \mathrm{H}) ;{ }^{13} \mathrm{C}$ NMR $\left(75 \mathrm{MHz}\right.$, benzene- $\left.d_{6}\right) \delta 174.1,138.0,128.9(2 \mathrm{C}), 128.5$ (2C), 127.8, 72.5, 71.6, 63.7, 53.8, 45.3, 34.5, 26.1 (3C), 26.0 (3C), 18.3, 18.2, -4.2, -4.4, -4.8, -5.0. Anal. $\left(\mathrm{C}_{25} \mathrm{H}_{43} \mathrm{NO}_{3} \mathrm{Si}_{2}\right): \mathrm{C}, \mathrm{H}, \mathrm{N}$.

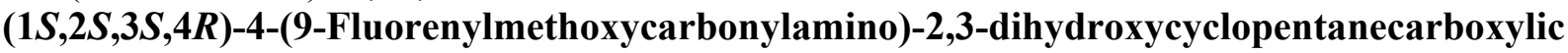
Acid (Fmoc-Acpca19-OH) (19). Anhydrous ammonia (45 mL) was condensed into a two-necked flask containing a solution of $\mathbf{1 7}(1.33 \mathrm{~g}, 2.88 \mathrm{mmol})$ in anhydrous THF $(65 \mathrm{~mL})$ maintained at $-78{ }^{\circ} \mathrm{C}$. 
Sodium metal was added to the mixture until the blue color persisted. The reaction was stirred for 30 min at $-78{ }^{\circ} \mathrm{C}$ monitoring by TLC, and quenched by addition of solid $\mathrm{NH}_{4} \mathrm{Cl}$. The ammonia was evaporated, the residue treated with saturated $\mathrm{NH}_{4} \mathrm{Cl}$ aqueous solution and extracted with EtOAc. The organic extracts were dried $\left(\mathrm{MgSO}_{4}\right)$, filtered, and concentrated in vacuo. Purification by flash chromatography (EtOAc/hexane 1:1) gave a $N$-deprotected bicyclic intermediate $(1.05 \mathrm{~g}, 98 \%)$ as a white solid: $\mathrm{mp} 70-73{ }^{\circ} \mathrm{C}$; $[\alpha]_{\mathrm{D}}{ }^{20}+4.3\left(c 2.1, \mathrm{CHCl}_{3}\right) ;{ }^{1} \mathrm{H} \mathrm{NMR}\left(300 \mathrm{MHz}, \mathrm{CDCl}_{3}\right) \delta 5.54(\mathrm{bs}, 1 \mathrm{H})$, $4.07(\mathrm{~d}, J=3.9 \mathrm{~Hz}, 1 \mathrm{H}), 3.71(\mathrm{~s}, 1 \mathrm{H}), 3.42(\mathrm{~s}, 1 \mathrm{H}), 2.62(\mathrm{bd}, J=3.9 \mathrm{~Hz}, 1 \mathrm{H}), 2.04(\mathrm{~m}, 2 \mathrm{H}), 0.89(\mathrm{~s}$, $18 \mathrm{H}), 0.12(\mathrm{~s}, 3 \mathrm{H}), 0.10(\mathrm{~s}, 3 \mathrm{H}), 0.09$ (s, 3H), 0.08 (s, 3H); ${ }^{13} \mathrm{C} \mathrm{NMR}\left(75 \mathrm{MHz}, \mathrm{CDCl}_{3}\right) \delta 177.3,81.7$, $80.2,58.9,49.8,37.2,25.7$ (6 C), 17.9, 17.8, $-4.5(2 \mathrm{C}),-4.4(2 \mathrm{C})$.

This bicyclic intermediate $(1.05 \mathrm{~g}, 2.82 \mathrm{mmol})$ was dissolved in $6 \mathrm{~N}$ aq $\mathrm{HCl}(10 \mathrm{~mL})$ and the resulting mixture was warmed to $80{ }^{\circ} \mathrm{C}$ for $5 \mathrm{~h}$. The reaction was then cooled to room temperature, diluted with water $(20 \mathrm{~mL})$, and washed with $\mathrm{CH}_{2} \mathrm{Cl}_{2}(3 \times 20 \mathrm{~mL})$. The aqueous phase was concentrated under vacuum to give a carbocyclic amino acid intermediate $(556 \mathrm{mg}$, quant.) as a white residue, which was used as such in the subsequent $N$-Fmoc protection: white solid, mp $196{ }^{\circ} \mathrm{C}(\mathrm{dec}) ;[\alpha]_{\mathrm{D}}{ }^{20}-29.4(c 0.3$, $\left.\mathrm{H}_{2} \mathrm{O}\right) ;{ }^{1} \mathrm{H}$ NMR $\left(300 \mathrm{MHz}, \mathrm{D}_{2} \mathrm{O}\right) \delta 4.15$ (ddd, $\left.J=6.0,4.6,0.8 \mathrm{~Hz}, 1 \mathrm{H}\right), 4.10(\mathrm{t}, J=4.6 \mathrm{~Hz}, 1 \mathrm{H}), 3.46$ (dddt, $J=9.2,7.1,4.4,0.8 \mathrm{~Hz}, 1 \mathrm{H}$ ), 2.99 (dddd, $J=8.5,7.7,6.1,0.8 \mathrm{~Hz}, 1 \mathrm{H}$ ), 2.38 (dt, $J=14.2,8.4$ $\mathrm{Hz}, 1 \mathrm{H}), 1.98(\mathrm{ddd}, J=14.1,6.3,5.7 \mathrm{~Hz}, 1 \mathrm{H}) ;{ }^{13} \mathrm{C} \mathrm{NMR}\left(75 \mathrm{MHz}, \mathrm{D}_{2} \mathrm{O}\right) \delta 183.4,83.0,80.5,58.8$, 51.6, 32.7 .

A stirring solution of this deprotected amino acid intermediate $(556 \mathrm{mg}, 2.82 \mathrm{mmol})$ (Acpca19$\mathbf{O H} \cdot \mathbf{H C l})$ in water $(10 \mathrm{~mL})$ was neutralized with $\mathrm{Et}_{3} \mathrm{~N}$ and 9-fluorenylmethyl- $N$-succinimidyl carbonate (FmocOSu, $951 \mathrm{mg}, 2.82 \mathrm{mmol}$ ) dissolved in acetonitrile $(4 \mathrm{~mL})$ was added at room temperature. The $\mathrm{pH}$ was maintained at $\mathrm{pH} 8.0$ using triethylamine. The solution was stirred for $4 \mathrm{~h}$ at room temperature. The organic phase was evaporated under vacuum and the residue was treated with 5 $\mathrm{mL}$ of $2 \%$ aqueous $\mathrm{HCl}$. The resulting heterogeneous mixture was extracted with EtOAc $(4 \times 15 \mathrm{~mL})$ and the organic layers were collected, dried, filtered, and concentrated to furnish the crude protected amino acid 19. Purification by flash chromatography (EtOAc/MeOH 7:3) gave the pure compound 19 (984 mg, 91\%) as a white foam: $[\alpha]_{\mathrm{D}}{ }^{20}-6.5(c 2.0, \mathrm{MeOH}) ;{ }^{1} \mathrm{H}$ NMR (400 MHz, $\left.\mathrm{CD}_{3} \mathrm{OD}\right) \delta 7.82(\mathrm{~d}, J$ $=7.2 \mathrm{~Hz}, 2 \mathrm{H}), 7.68(\mathrm{~d}, J=7.6 \mathrm{~Hz}, 2 \mathrm{H}), 7.42(\mathrm{t}, J=7.2 \mathrm{~Hz}, 2 \mathrm{H}), 7.34$ (t, $J=7.2 \mathrm{~Hz}, 2 \mathrm{H}), 4.37(\mathrm{~d}, J=$ $6.9 \mathrm{~Hz}, 2 \mathrm{H}), 4.28(\mathrm{t}, J=6.8 \mathrm{~Hz}, 1 \mathrm{H}), 4.11(\mathrm{dd}, J=6.0,3.6 \mathrm{~Hz}, 1 \mathrm{H}), 3.89$ (t, $J=4.8 \mathrm{~Hz}, 1 \mathrm{H}), 3.81(\mathrm{td}, J$ $=8.4,4.8 \mathrm{~Hz}, 1 \mathrm{H}), 3.04(\mathrm{td}, J=9.0,6.8 \mathrm{~Hz}, 1 \mathrm{H}), 2.33(\mathrm{dt}, J=13.2,8.4 \mathrm{~Hz}, 1 \mathrm{H}), 2.01(\mathrm{dt}, J=13.6,9.2$ $\mathrm{Hz}, 1 \mathrm{H}) ;{ }^{13} \mathrm{C}$ NMR $\left(100 \mathrm{MHz}, \mathrm{CD}_{3} \mathrm{OD}\right) \delta 177.5,158.6,145.3(2 \mathrm{C}), 142.6(2 \mathrm{C}), 128.8(2 \mathrm{C}), 128.1$ (2C), $126.2(2 \mathrm{C}), 120.9(2 \mathrm{C}), 83.9,78.3,67.8,58.0,47.4,32.1,26.3$. Anal. $\left(\mathrm{C}_{21} \mathrm{H}_{21} \mathrm{NO}_{6}\right): \mathrm{C}, \mathrm{H}, \mathrm{N}$.

(1S,2R,3S,4R)-4-(9-Fluorenylmethoxycarbonylamino)-2,3-dihydroxycyclopentanecarboxylic

Acid (Fmoc-Acpca20-OH) (20). Compound 20 was obtained from bicyclic lactam 18 (0.34 g, 0.74 mmol) by adopting the three-step procedure to 19.

$N$-deprotected bicyclic intermediate (266 mg, 97\%): a colorless oil, $[\alpha]_{\mathrm{D}}{ }^{20}-40.6\left(\mathrm{c} 1.7, \mathrm{CHCl}_{3}\right) ;{ }^{1} \mathrm{H}$ NMR $\left(300 \mathrm{MHz}, \mathrm{CDCl}_{3}\right) \delta 6.22(\mathrm{bs}, 1 \mathrm{H}), 4.06(\mathrm{dd}, J=5.4,1.2 \mathrm{~Hz}, 1 \mathrm{H}), 3.90(\mathrm{bd}, J=5.7 \mathrm{~Hz}, 1 \mathrm{H})$, $3.52(\mathrm{bs}, 1 \mathrm{H}), 2.46(\mathrm{bs}, 1 \mathrm{H}), 2.28(\mathrm{dd}, J=9.6,1.2 \mathrm{~Hz}, 1 \mathrm{H}), 1.95(\mathrm{dt}, J=9.9,1.8 \mathrm{~Hz}, 1 \mathrm{H}), 0.90(\mathrm{~s}, 18 \mathrm{H})$, $0.12(\mathrm{~s}, 3 \mathrm{H}), 0.09(\mathrm{~s}, 3 \mathrm{H}), 0.08(\mathrm{~s}, 3 \mathrm{H}), 0.07(\mathrm{~s}, 3 \mathrm{H}) ;{ }^{13} \mathrm{C} \mathrm{NMR}\left(75 \mathrm{MHz}, \mathrm{CDCl}_{3}\right) \delta 179.2,74.1,70.2$, $59.9,52.5,35.9,25.9(6 \mathrm{C}), 18.2(2 \mathrm{C}),-4.4(2 \mathrm{C}),-4.9(2 \mathrm{C})$.

Deprotected amino acid intermediate (142 mg, quant.) (Acpca20-OH·HCI): white solid, $\mathrm{mp} 150{ }^{\circ} \mathrm{C}$ (dec.); $[\alpha]_{\mathrm{D}}{ }^{20}-5.4\left(c 0.2, \mathrm{H}_{2} \mathrm{O} / \mathrm{MeOH} 4: 1 \mathrm{v} / \mathrm{v}\right) ;{ }^{1} \mathrm{H} \mathrm{NMR}\left(300 \mathrm{MHz}, \mathrm{D}_{2} \mathrm{O}\right) \delta 4.16(\mathrm{dd}, J=6.2,4.6 \mathrm{~Hz}$, $1 \mathrm{H}), 4.11(\mathrm{t}, J=4.5 \mathrm{~Hz}, 1 \mathrm{H}), 3.47(\mathrm{ddd}, J=8.3,6.4,4.8 \mathrm{~Hz}, 1 \mathrm{H}), 3.03(\mathrm{td}, J=8.0,6.4 \mathrm{~Hz}, 1 \mathrm{H}), 2.39$ $(\mathrm{dt}, J=14.2,8.4 \mathrm{~Hz}, 1 \mathrm{H}), 2.00(\mathrm{dt}, J=14.2,7.3 \mathrm{~Hz}, 1 \mathrm{H}) ;{ }^{13} \mathrm{C} \mathrm{NMR}\left(75 \mathrm{MHz}, \mathrm{D}_{2} \mathrm{O}\right) \delta 180.0,80.0$, $77.4,55.8,48.5,29.7$.

Compound $20\left(190 \mathrm{mg}, 69 \%, 67 \%\right.$ for the three steps from 18): white solid, $[\alpha]_{\mathrm{D}}{ }^{20}-2.8(c 1.4$, $\mathrm{MeOH}) ;{ }^{1} \mathrm{H}$ NMR $\left(400 \mathrm{MHz}, \mathrm{CD}_{3} \mathrm{OD}\right) \delta 7.77(\mathrm{~d}, J=7.2 \mathrm{~Hz}, 2 \mathrm{H}), 7.64(\mathrm{~d}, J=7.6 \mathrm{~Hz}, 2 \mathrm{H}), 7.37(\mathrm{t}, J=$ $7.2 \mathrm{~Hz}, 2 \mathrm{H}), 7.29(\mathrm{t}, J=7.2 \mathrm{~Hz}, 2 \mathrm{H}), 4.33(\mathrm{~d}, J=6.8 \mathrm{~Hz}, 2 \mathrm{H}), 4.18(\mathrm{~m}, 2 \mathrm{H}), 3.90(\mathrm{q}, J=6.8 \mathrm{~Hz}, 1 \mathrm{H})$, 
$3.79(\mathrm{t}, J=6.0 \mathrm{~Hz}, 1 \mathrm{H}), 2.80(\mathrm{td}, J=8.4,5.6 \mathrm{~Hz}, 1 \mathrm{H}), 2.40(\mathrm{dt}, J=13.2,8.4 \mathrm{~Hz}, 1 \mathrm{H}), 1.61(\mathrm{dt}, J=$ $12.8,8.4 \mathrm{~Hz}, 1 \mathrm{H}) ;{ }^{13} \mathrm{C} \mathrm{NMR}\left(100 \mathrm{MHz}, \mathrm{CD}_{3} \mathrm{OD}\right) \delta 174.9,158.6,145.3$ (2C), $142.6(2 \mathrm{C}), 128.7(2 \mathrm{C})$, 128.1 (2C), 126.2 (2C), 120.9 (2C), 77.8, 75.0, 67.7, 57.1, 48.4, 31.9, 26.3. Anal. $\left(\mathrm{C}_{21} \mathrm{H}_{21} \mathrm{NO}_{6}\right): \mathrm{C}, \mathrm{H}$, $\mathrm{N}$.

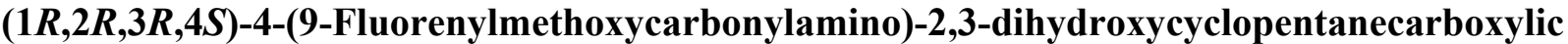
Acid (Fmoc-Acpca21-OH) (21) and (1R,2S,3R,4S)-4-(9-Fluorenylmethoxycarbonylamino)-2,3dihydroxycyclopentanecarboxylic Acid (Fmoc-Acpca22-OH) (22). The title compounds were prepared from silyloxypyrrole $12(1.38 \mathrm{~g}, 4.66 \mathrm{mmol})$ and L-glyceraldehyde $(S$-13) $(728 \mathrm{mg}, 5.59$ mmol) according to the ten-step procedure described for compounds 19 and 20. All intermediate compounds proved identical in all respects to the enantiomeric counterparts previously described (compounds 14-18) except for the optical rotations, identical in absolute value and of opposite sign.

Compound 21 (536 mg, 30\% over ten steps): a white foam, $[\alpha]_{\mathrm{D}}{ }^{20}+6.3(c 2.1, \mathrm{MeOH}) ;{ }^{1} \mathrm{H}$ and ${ }^{13} \mathrm{C}$ NMR identical to the enantiomeric counterpart 19. Anal. $\left(\mathrm{C}_{21} \mathrm{H}_{21} \mathrm{NO}_{6}\right): \mathrm{C}, \mathrm{H}, \mathrm{N}$.

Compound 22 (250 mg, 14\% over ten steps): a white foam, $[\alpha]_{\mathrm{D}}{ }^{20}+2.5(c 2.1, \mathrm{MeOH}) ;{ }^{1} \mathrm{H}$ and ${ }^{13} \mathrm{C}$ NMR identical to the enantiomeric counterpart 20. Anal. $\left(\mathrm{C}_{21} \mathrm{H}_{21} \mathrm{NO}_{6}\right)$ : C, H, N.

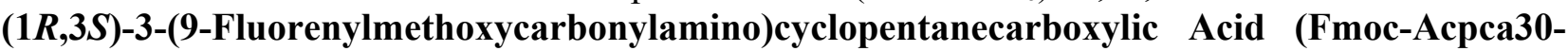
OH) (30). Commercial (1R,3S)-3-aminocyclopentanecarboxylic acid 23 (200 $\mathrm{mg}, 1.55 \mathrm{mmol})$ dissolved in water $(5 \mathrm{~mL})$ was neutralized with triethylamine and FmocOSu $(523 \mathrm{mg}, 1.55 \mathrm{mmol})$, dissolved in acetonitrile $(2 \mathrm{~mL})$, was added at room temperature. The $\mathrm{pH}$ was maintained at $\mathrm{pH} 8.0$ using triethylamine. The solution was stirred for $2 \mathrm{~h}$ at room temperature. The organic phase was then evaporated under vacuum and the water residue was treated with $1 \mathrm{~mL}$ of $2 \%$ aqueous $\mathrm{HCl}$. The resulting heterogeneous mixture was extracted with EtOAc $(4 \times 5 \mathrm{~mL})$ and the organic layers were collected, dried, filtered, and concentrated to furnish a crude residue which was purified by flash chromatography (EtOAc). Amino acid 30 (408 mg, 75\%) was obtained as a white solid, mp 164$166^{\circ} \mathrm{C},[\alpha]_{\mathrm{D}}^{20}-8.4(c 4.1, \mathrm{MeOH}) ;{ }^{1} \mathrm{H}$ NMR $\left(400 \mathrm{MHz}, \mathrm{CD}_{3} \mathrm{OD}\right) \delta 7.78(\mathrm{~d}, J=7.6 \mathrm{~Hz}, 2 \mathrm{H}), 7.63(\mathrm{~d}, J=$ $7.6 \mathrm{~Hz}, 2 \mathrm{H}), 7.38(\mathrm{t}, J=7.2 \mathrm{~Hz}, 2 \mathrm{H}), 7.30(\mathrm{t}, J=7.2 \mathrm{~Hz}, 2 \mathrm{H}), 4.32(\mathrm{~d}, J=6.4 \mathrm{~Hz}, 2 \mathrm{H}), 4.18(\mathrm{t}, J=6.4$ $\mathrm{Hz}, 1 \mathrm{H}), 3.95(\mathrm{~m}, 1 \mathrm{H}), 2.80(\mathrm{~m}, 1 \mathrm{H}), 2.24(\mathrm{dt}, J=12.4,8.0 \mathrm{~Hz}, 1 \mathrm{H}), 1.94(\mathrm{~m}, 3 \mathrm{H}), 1.71(\mathrm{dt}, J=12.8$, $8.0 \mathrm{~Hz}, 1 \mathrm{H}), 1.60(\mathrm{~m}, 1 \mathrm{H}) ;{ }^{13} \mathrm{C} \mathrm{NMR}\left(100 \mathrm{MHz}, \mathrm{CD}_{3} \mathrm{OD}\right) \delta 180.1,158.3,145.4(2 \mathrm{C}), 142.6(2 \mathrm{C})$, 128.7 (2C), 128.1 (2C), 126.2 (2C), 120.9 (2C), 67.6, 53.7, 48.4, 43.3, 37.2, 33.2, 28.5. Anal. $\left(\mathrm{C}_{21} \mathrm{H}_{21} \mathrm{NO}_{4}\right): \mathrm{C}, \mathrm{H}, \mathrm{N}$.

(1S,3R)-3-(9-Fluorenylmethoxycarbonylamino)cyclopentanecarboxylic Acid (Fmoc-Acpca31OH) (31). The title compound was prepared from commercial $(1 S, 3 R)$-3-aminocyclopentanecarboxylic acid (24) (200 mg, $1.55 \mathrm{mmol}$ ) according to the procedure described for 30. After flash chromatographic purification (EtOAc), amino acid $\mathbf{3 1}$ was obtained (207 $\mathrm{mg}, 38 \%$ ) as a white solid, $\mathrm{mp} 166-168^{\circ} \mathrm{C} ;[\alpha]_{\mathrm{D}}{ }^{20}+11.6(c 2.1, \mathrm{MeOH}) ;{ }^{1} \mathrm{H}$ and ${ }^{13} \mathrm{C}$ NMR identical to the enantiomeric counterpart 30. Anal. $\left(\mathrm{C}_{21} \mathrm{H}_{21} \mathrm{NO}_{4}\right)$ : $\mathrm{C}, \mathrm{H}, \mathrm{N}$.

(1R,3R)-3-(9-Fluorenylmethoxycarbonylamino)cyclopentanecarboxylic Acid (Fmoc-Acpca32OH) (32). Commercial (1R,3R)-1-(tert-butoxycarbonylamino)cyclopentane-3-carboxylic acid (25) $(200 \mathrm{mg}, 0.87 \mathrm{mmol})$ was dissolved in $6 \mathrm{~N}$ aq $\mathrm{HCl}(5 \mathrm{~mL})$ and the resulting mixture was warmed to $80^{\circ} \mathrm{C}$ for $5 \mathrm{~h}$. The reaction was cooled to room temperature, diluted with water $(5 \mathrm{~mL})$, and washed with $\mathrm{CH}_{2} \mathrm{Cl}_{2}(3 \times 5 \mathrm{~mL})$. The aqueous phase was concentrated under vacuum to give a deprotected amino acid intermediate $\left(144 \mathrm{mg}\right.$, quant.) as a white solid, $\mathrm{mp} 117-120^{\circ} \mathrm{C},[\alpha]_{\mathrm{D}}{ }^{20}-9.3$ (c 3.2 , $\mathrm{MeOH} / \mathrm{H}_{2} \mathrm{O}$ 6:4); which was used as such in the subsequent $N$-Fmoc protection.

The $N$-Fmoc protection of this amino acid intermediate $(144 \mathrm{mg}, 0.87 \mathrm{mmol})$ was carried out following the procedure described for 30. After flash chromatographic purification $(\mathrm{EtOAc} / \mathrm{MeOH}$ 9:1), amino acid 32 was obtained (153 $\mathrm{mg}, 50 \%)$ as a white solid, mp $142-145{ }^{\circ} \mathrm{C} ;[\alpha]_{\mathrm{D}}{ }^{20}-6.5(c 0.6$, $\mathrm{MeOH}) ;{ }^{1} \mathrm{H}$ NMR $\left(400 \mathrm{MHz}, \mathrm{CD}_{3} \mathrm{OD}\right) \delta 7.78(\mathrm{~d}, J=7.6 \mathrm{~Hz}, 2 \mathrm{H}), 7.64(\mathrm{~d}, J=6.8 \mathrm{~Hz}, 2 \mathrm{H}), 7.38(\mathrm{t}, J=$ $7.6 \mathrm{~Hz}, 2 \mathrm{H}), 7.31(\mathrm{t}, J=7.6 \mathrm{~Hz}, 2 \mathrm{H}), 4.35(\mathrm{~d}, J=6.8 \mathrm{~Hz}, 2 \mathrm{H}), 4.17(\mathrm{t}, J=6.4 \mathrm{~Hz}, 1 \mathrm{H}), 4.04(\mathrm{~m}, 1 \mathrm{H})$, $2.93(\mathrm{~m}, 1 \mathrm{H}), 2.15(\mathrm{dt}, J=12.8,7.2 \mathrm{~Hz}, 1 \mathrm{H}), 2.04(\mathrm{~m}, 2 \mathrm{H}), 1.82(\mathrm{~m}, 2 \mathrm{H}), 1.56(\mathrm{~m}, 1 \mathrm{H}) ;{ }^{13} \mathrm{C}$ NMR $(100$ 
$\left.\mathrm{MHz}, \mathrm{CD}_{3} \mathrm{OD}\right) \delta 179.7,158.3,145.3(2 \mathrm{C}), 142.6(2 \mathrm{C}), 128.7$ (2C), 128.1 (2C), $126.1(2 \mathrm{C}), 120.9(2 \mathrm{C})$, 67.4, 53.5, 48.5, 43.0, 37.2, 33.4, 28.9. Anal. $\left(\mathrm{C}_{21} \mathrm{H}_{21} \mathrm{NO}_{4}\right): \mathrm{C}, \mathrm{H}, \mathrm{N}$.

(1S,3S)-3-(9-Fluorenylmethoxycarbonylamino)cyclopentanecarboxylic Acid (Fmoc-Acpca33OH) (33). The title compound was prepared from commercial (1S,3S)-1-(tertbutoxycarbonylamino)cyclopentane-3-carboxylic acid methyl ester (26) (200 mg, $0.82 \mathrm{mmol}$ ) according to the two-step procedure described for 32. After flash chromatographic purification (EtOAc/MeOH 9:1), protected amino acid 33 was obtained (196 mg, 68\%) as a white solid, mp 142$145{ }^{\circ} \mathrm{C} ;[\alpha]_{\mathrm{D}}{ }^{20}+6.6(c 0.8, \mathrm{MeOH}) ;{ }^{1} \mathrm{H}$ and ${ }^{13} \mathrm{C}$ NMR identical to the enantiomeric counterpart 32. Anal. $\left(\mathrm{C}_{21} \mathrm{H}_{21} \mathrm{NO}_{4}\right)$ : C, H, N.

$(1 S, 3 S, 4 S)$-3-(9-Fluorenylmethoxycarbonylamino)-4-hydroxycyclopentanecarboxylic Acid (Fmoc-Acpca34-OH) (34). The title compound was prepared from commercial $(1 S, 2 S, 4 S)$-1-(tertbutoxycarbonylamino)-2-hydroxycyclopentane-4-carboxylic acid methyl ester (27) (300 mg, 1.16 $\mathrm{mmol}$ ) according to the two-step procedure described for 32. After flash chromatographic purification (EtOAc/MeOH 9:1), protected amino acid 34 was obtained $\left(366 \mathrm{mg}, 86 \%\right.$ ) as a glassy solid, $[\alpha]_{\mathrm{D}}{ }^{20}+1.3$ (c 1.6, MeOH); ${ }^{1} \mathrm{H}$ NMR (400 MHz, $\left.\mathrm{CD}_{3} \mathrm{OD}\right) \delta 7.80(\mathrm{~d}, J=7.6 \mathrm{~Hz}, 2 \mathrm{H}), 7.67(\mathrm{~d}, J=7.2 \mathrm{~Hz}, 2 \mathrm{H}), 7.40$ $(\mathrm{t}, J=7.2 \mathrm{~Hz}, 2 \mathrm{H}), 7.32(\mathrm{t}, J=7.6 \mathrm{~Hz}, 2 \mathrm{H}), 4.38(\mathrm{~d}, J=7.2 \mathrm{~Hz}, 2 \mathrm{H}), 4.20(\mathrm{t}, J=6.8 \mathrm{~Hz}, 1 \mathrm{H}), 3.99(\mathrm{q}, J$ $=6.0 \mathrm{~Hz}, 1 \mathrm{H}), 3.83(\mathrm{~m}, 1 \mathrm{H}), 2.94$ (quint, $J=8.0 \mathrm{~Hz}, 1 \mathrm{H}), 2.35(\mathrm{dt}, J=13.2,7.6 \mathrm{~Hz}, 1 \mathrm{H}), 2.28$ (ddd, $J=$ $15.2,8.8,6.4 \mathrm{~Hz}, 1 \mathrm{H}), 1.86(\mathrm{dt}, J=13.2,7.6 \mathrm{~Hz}, 1 \mathrm{H}), 1.79(\mathrm{ddd}, J=14.8,9.6,6.4 \mathrm{~Hz}, 1 \mathrm{H}) ;{ }^{13} \mathrm{C} \mathrm{NMR}$ $\left(100 \mathrm{MHz}, \mathrm{CD}_{3} \mathrm{OD}\right) \delta 179.5,158.6,145.3(2 \mathrm{C}), 142.6(2 \mathrm{C}), 128.8(2 \mathrm{C}), 128.1(2 \mathrm{C}), 126.2(2 \mathrm{C}), 120.9$ (2C), 77.6, 67.5, 59.8, 48.5, 40.2, 36.6, 33.5. Anal. $\left(\mathrm{C}_{21} \mathrm{H}_{21} \mathrm{NO}_{5}\right): \mathrm{C}, \mathrm{H}, \mathrm{N}$.

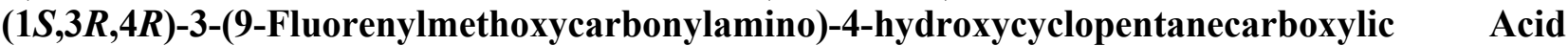
(Fmoc-Acpca35-OH) (35). The title compound was prepared from commercial $(1 R, 2 R, 4 S)-1-($ tertbutoxycarbonylamino)-2-hydroxycyclopentane-4-carboxylic acid methyl ester (28) (250 mg, 0.96 mmol) according to the two-step procedure described for 32. After flash chromatographic purification (EtOAc/MeOH 8:2), protected amino acid 35 was obtained $(312 \mathrm{mg}, 88 \%)$ as a glassy solid, $[\alpha]_{\mathrm{D}}{ }^{20}-2.3$ $(c 1.8, \mathrm{MeOH}) ;{ }^{1} \mathrm{H}$ NMR $\left(400 \mathrm{MHz}, \mathrm{CD}_{3} \mathrm{OD}\right) \delta 7.77(\mathrm{~d}, J=7.2 \mathrm{~Hz}, 2 \mathrm{H}), 7.64(\mathrm{~d}, J=7.6 \mathrm{~Hz}, 2 \mathrm{H}), 7.37$ $(\mathrm{t}, J=7.2 \mathrm{~Hz}, 2 \mathrm{H}), 7.29(\mathrm{t}, J=7.6 \mathrm{~Hz}, 2 \mathrm{H}), 4.32(\mathrm{~d}, J=7.2 \mathrm{~Hz}, 2 \mathrm{H}), 4.18(\mathrm{t}, J=6.8 \mathrm{~Hz}, 1 \mathrm{H}), 4.02(\mathrm{~m}$, $1 \mathrm{H}), 3.78(\mathrm{~m}, 1 \mathrm{H}), 2.97$ (quint, $J=8.0 \mathrm{~Hz}, 1 \mathrm{H}$ ), 2.345 (dt, $J=12.8,8.8 \mathrm{~Hz}, 1 \mathrm{H}$ ), 2.13 (dt, $J=14.4,6.8$ $\mathrm{Hz}, 1 \mathrm{H}), 1.88(\mathrm{ddd}, J=13.2,9.2,4.4 \mathrm{~Hz}, 1 \mathrm{H}), 1.71(\mathrm{dt}, J=13.2,8.0 \mathrm{~Hz}, 1 \mathrm{H}) ;{ }^{13} \mathrm{C} \mathrm{NMR}(100 \mathrm{MHz}$, $\left.\mathrm{CD}_{3} \mathrm{OD}\right) \delta 181.1,158.5,145.3$ (2C), 142.6 (2C), 128.7 (2C), 128.1 (2C), 126.2 (2C), 120.9 (2C), 77.7, 67.7, 60.5, 48.4, 41.6, 37.0, 34.5. Anal. $\left(\mathrm{C}_{21} \mathrm{H}_{21} \mathrm{NO}_{5}\right): \mathrm{C}, \mathrm{H}, \mathrm{N}$.

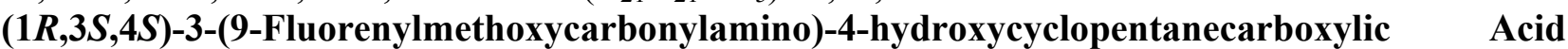
(Fmoc-Acpca36-OH) (36). The title compound was prepared from commercial $(1 S, 2 S, 4 R)$-1-(tertbutoxycarbonylamino)-2-hydroxycyclopentane-4-carboxylic acid methyl ester (29) (122 mg, 0.47 mmol) according to the two-step procedure described for 32. After flash chromatographic purification (EtOAc/MeOH 8:2), protected amino acid 36 was obtained $\left(144 \mathrm{mg}, 83 \%\right.$ ) as a glassy solid, $[\alpha]_{\mathrm{D}}{ }^{20}+2.2$ (c 1.4, MeOH); ${ }^{1} \mathrm{H}$ and ${ }^{13} \mathrm{C}$ NMR identical to the enantiomeric counterpart 35. Anal. $\left(\mathrm{C}_{21} \mathrm{H}_{21} \mathrm{NO}_{5}\right)$ : $\mathrm{C}$, $\mathrm{H}, \mathrm{N}$. 
Table S1. ${ }^{1} \mathrm{H}$ and ${ }^{13} \mathrm{C}$ NMR chemical shifts (ppm) in $\mathrm{H}_{2} \mathrm{O}$ at $300 \mathrm{~K}$, temperature coefficients for the amide protons $(\mathrm{ppb} / \mathrm{K})$, and coupling constants ${ }^{3} J_{\mathrm{NH}-\alpha \mathrm{H}}(\mathrm{Hz})$ of cyclopeptides 1-4. Conditions, see Experimental Section.

\begin{tabular}{|c|c|c|c|c|c|c|c|c|c|c|c|c|c|}
\hline & Residue & $\mathrm{NH}$ & $\Delta \delta / \Delta \mathrm{T}$ & $\mathrm{H} / \mathrm{H}_{2}(\alpha)$ & $\mathrm{H}_{2}(\beta)$ & $\mathrm{H}_{2}(\gamma)$ & $\mathrm{H}_{2}(\delta)$ & $\mathrm{HN}(\varepsilon)$ & ${ }^{3} J_{\mathrm{NH}-\alpha \mathrm{H}}$ & $C(\alpha)$ & $C(\beta)$ & $\mathrm{C}(\gamma)$ & $C(\delta)$ \\
\hline \multirow[t]{5}{*}{ Compd 1} & Arg & 8.57 & -5.34 & 4.03 & 1.82 & $1.67,1.61$ & 3.21 & 7.18 & 6.90 & 57.73 & 29.40 & 27.50 & 43.50 \\
\hline & Gly & 8.57 & -5.30 & $4.05,3.68$ & & & & & & 46.76 & & & \\
\hline & Asp & 8.36 & -6.20 & 4.50 & $2.75,2.70$ & & & & 8.40 & 55.10 & 40.62 & & \\
\hline & Acpca 19 & \multicolumn{11}{|c|}{$7.58(\mathrm{NH}), 4.25(\mathrm{H} 5), 4.248(\mathrm{H} 4), 3.95(\mathrm{H} 3), 3.07(\mathrm{H1}), 2.26,1.92(\mathrm{H} 2),-\Delta \delta / \Delta \mathrm{T}=-3.5,{ }^{3} J_{\mathrm{NH}-\alpha \mathrm{H}}=9.10$} & \\
\hline & & \multicolumn{11}{|c|}{85.80 (C5), $82.00(\mathrm{C} 4), 58.20(\mathrm{C} 3), 49.04(\mathrm{C} 1), 34.50(\mathrm{C} 2)$} & \\
\hline \multirow[t]{5}{*}{ Compd 2} & Arg & 8.45 & -5.7 & 4.12 & 1.85 & $1.74,1.64$ & 3.24 & 7.20 & 3.90 & 59.20 & 28.83 & 27.30 & 43.47 \\
\hline & Gly & 8.57 & -5.5 & $4.07,3.76$ & & & & & & 46.70 & & & \\
\hline & Asp & 8.32 & -2.5 & 4.68 & 2.87 & & & & 8.33 & 52.91 & 37.76 & & \\
\hline & Acpca 20 & \multicolumn{12}{|c|}{$7.56(\mathrm{NH}), 4.24(\mathrm{H} 5), 4.05(\mathrm{H} 4), 4.00(\mathrm{H} 3), 2.94(\mathrm{H1}), 2.39,2.06(\mathrm{H} 2), \Delta \delta / \Delta \mathrm{T}=-3.2,{ }^{3} J_{\mathrm{NH}-\alpha \mathrm{H}}=5.40$} \\
\hline & & \multicolumn{11}{|c|}{79.25 (C5), 78.05 (C4), 59.74 (C3), 51.35 (C1), 30.77 (C2) } & \\
\hline \multirow[t]{5}{*}{ Compd 3} & Arg & 8.46 & -4.0 & 4.09 & 1.79 & $1.73,1.63$ & 3.23 & 7.19 & 8.80 & 58.02 & 29.21 & 27.30 & 43.40 \\
\hline & Gly & 8.70 & -5.5 & $4.11,3.65$ & & & & & & 46.84 & & & \\
\hline & Asp & 8.45 & -3.0 & 4.75 & $2.91,2.86$ & & & & 8.80 & 52.73 & 37.51 & & \\
\hline & Acpca 21 & \multicolumn{12}{|c|}{$7.48(\mathrm{NH}), 4.45(\mathrm{H} 5), 4.10(\mathrm{H} 4), 4.06(\mathrm{H} 3), 3.11(\mathrm{H} 1), 2.44,1.69(\mathrm{H} 2),-\Delta \delta / \Delta \mathrm{T}=-1.0^{3} J_{\mathrm{NH}-\alpha \mathrm{H}}=8.60$} \\
\hline & & \multicolumn{12}{|c|}{87.09 (C5), 83.35 (C4), 58.62 (C3), 49.95 (C1), 35.37 (C2) } \\
\hline \multirow[t]{5}{*}{ Compd 4} & Arg & 8.42 & -4.6 & 4.11 & 1.80 & $1.70,1.62$ & 3.24 & 7.19 & 8.80 & 57.71 & 29.14 & 27.30 & 43.49 \\
\hline & Gly & 8.63 & -5.0 & $4.13,3.60$ & & & & & & 46.72 & & & \\
\hline & Asp & 8.42 & -3.1 & 4.68 & $2.91,2.84$ & & & & 8.68 & 52.73 & 37.59 & & \\
\hline & Acpca 22 & \multicolumn{12}{|c|}{$7.33(\mathrm{NH}), 4.43(\mathrm{H} 5), 4.31(\mathrm{H} 4), 4.06(\mathrm{H} 3), 2.97(\mathrm{H} 1), 2.68,1.78(\mathrm{H} 2), \Delta \delta / \Delta \mathrm{T}=-1.7,{ }^{3} J_{\mathrm{NH}-\alpha \mathrm{H}}=7.21$} \\
\hline & & \multicolumn{12}{|c|}{76.67 (C5), $82.80(\mathrm{C} 4), 59.81(\mathrm{C} 3), 52.46(\mathrm{C} 1), 36.31(\mathrm{C} 2)$} \\
\hline
\end{tabular}


Table S2. ${ }^{1} \mathrm{H}$ and ${ }^{13} \mathrm{C}$ NMR chemical shifts (ppm) in $\mathrm{H}_{2} \mathrm{O}$ at $300 \mathrm{~K}$, temperature coefficients for the amide protons $(\mathrm{ppb} / \mathrm{K})$, and coupling constants ${ }^{3} J_{\mathrm{NH}-\alpha \mathrm{H}}(\mathrm{Hz})$ of cyclopeptides 5-7. Conditions, see Experimental Section.

\begin{tabular}{|c|c|c|c|c|c|c|c|c|c|c|c|c|c|}
\hline & Residue & $\mathrm{NH}$ & $\Delta \delta / \Delta \mathrm{T}$ & $\mathrm{H} / \mathrm{H}_{2}(\alpha)$ & $\mathrm{H}_{2}(\beta)$ & $\mathrm{H}_{2}(\gamma)$ & $\mathrm{H}_{2}(\delta)$ & $\mathrm{HN}(\varepsilon)$ & ${ }^{3} J_{\mathrm{NH}-\alpha \mathrm{H}}$ & $C(\alpha)$ & $C(\beta)$ & $\mathrm{C}(\gamma)$ & $\mathrm{C}(\delta)$ \\
\hline \multirow[t]{5}{*}{ Compd $\mathbf{5}$} & Arg & 8.43 & -5.1 & 4.05 & 1.81 & $1.72,1.61$ & 3.24 & 7.19 & 4.1 & 59.00 & 28.90 & 27.30 & 43.39 \\
\hline & Gly & 8.56 & -5.1 & $4.07,3.74$ & & & & & & 46.83 & & & \\
\hline & Asp & 8.22 & -2.2 & 4.66 & 2.87 & & & & 8.5 & $*$ & 37.90 & & \\
\hline & Acpca 35 & \multicolumn{12}{|c|}{$7.55(\mathrm{NH}), 4.15(\mathrm{H} 4), 3.96(\mathrm{H} 3), 3.25(\mathrm{H} 1), 2.13,2.08(\mathrm{H} 5), 2.15,1.91(\mathrm{H} 2), \Delta \delta / \Delta \mathrm{T}=-3.0,{ }^{3} J_{\mathrm{NH}-\alpha \mathrm{H}}=5.6$} \\
\hline & & \multicolumn{11}{|c|}{$78.53(\mathrm{C} 4), 61.19(\mathrm{C} 3), 43.29(\mathrm{C} 1), 39.53(\mathrm{C} 5), 37.78(\mathrm{C} 2)$} & \\
\hline \multirow[t]{5}{*}{ Compd 6} & $\operatorname{Arg}$ & 8.49 & -5.4 & 4.05 & 1.81 & $1.72,1.63$ & 3.25 & 7.21 & 5.3 & 58.00 & 29.10 & 27.30 & 43.52 \\
\hline & Gly & 8.71 & -5.3 & $4.21,3.59$ & & & & & & 46.83 & & & \\
\hline & Asp & 8.32 & -2.0 & 4.70 & 2.88 & & & & 8.5 & * & 37.87 & & \\
\hline & Acpca 36 & \multicolumn{12}{|c|}{$7.28(\mathrm{NH}), 4.35(\mathrm{H} 4), 4.06(\mathrm{H} 3), 3.19(\mathrm{H} 1), 2.44,1.92(\mathrm{H} 2), 2.40,1.74(\mathrm{H} 5), \Delta \delta / \Delta \mathrm{T}=-1.3,{ }^{3} J_{\mathrm{NH}-\alpha \mathrm{H}}=7.6$} \\
\hline & & \multicolumn{12}{|c|}{$81.85(\mathrm{C} 4), 61.35(\mathrm{C} 3), 45.14(\mathrm{C} 1), 38.13(\mathrm{C} 5), 37.34(\mathrm{C} 2)$} \\
\hline \multirow[t]{5}{*}{ Compd 7} & $\operatorname{Arg}$ & 8.35 & -4.5 & 4.40 & $1.89,1.78$ & 1.64 & 3.22 & 7.24 & 7.4 & 55.90 & 31.14 & 27.17 & 43.33 \\
\hline & Gly & 8.57 & -4.8 & $3.99,3.58$ & & & & & & 45.81 & & & \\
\hline & Asp & 8.38 & -6.2 & 4.63 & 2.87 & & & & 7.4 & $*$ & 38.24 & & \\
\hline & Acpca 34 & \multicolumn{12}{|c|}{$7.67(\mathrm{NH}), 4.11(\mathrm{H} 4), 4.04(\mathrm{H} 3), 3.01(\mathrm{H} 1), 2.19,1.90(\mathrm{H} 2), 2.22,1.77(\mathrm{H} 5), \Delta \delta / \Delta \mathrm{T}=-2.3,{ }^{3} J_{\mathrm{NH}-\alpha \mathrm{H}}=7.2$} \\
\hline & & \multicolumn{12}{|c|}{$79.08(\mathrm{C} 4), 60.57(\mathrm{C} 3), 43.40(\mathrm{C} 1), 38.12(\mathrm{C} 5), 36.04(\mathrm{C} 2)$} \\
\hline
\end{tabular}

\footnotetext{
* Resonances not observed due to water resonance overlap.
} 
Table S3. ${ }^{1} \mathrm{H}$ and ${ }^{13} \mathrm{C}$ NMR chemical shifts (ppm) in $\mathrm{H}_{2} \mathrm{O}$ at $300 \mathrm{~K}$, temperature coefficients for the amide protons $(\mathrm{ppb} / \mathrm{K})$, and coupling constants ${ }^{3} J_{\mathrm{NH}-\alpha \mathrm{H}}(\mathrm{Hz})$ of cyclopeptides 8-11. Conditions, see Experimental Section.

\begin{tabular}{|c|c|c|c|c|c|c|c|c|c|c|c|c|c|}
\hline & Residue & $\mathrm{NH}$ & $\Delta \delta / \Delta \mathrm{T}$ & $\mathrm{H} / \mathrm{H}_{2}(\alpha)$ & $\mathrm{H}_{2}(\beta)$ & $\mathrm{H}_{2}(\gamma)$ & $\mathrm{H}_{2}(\delta)$ & $\mathrm{HN}(\varepsilon)$ & ${ }^{3} J_{\mathrm{NH}-\alpha \mathrm{H}}$ & $C(\alpha)$ & $C(\beta)$ & $\mathrm{C}(\gamma)$ & $C(\delta)$ \\
\hline \multirow[t]{5}{*}{ Compd 8} & Arg & 8.36 & -6.0 & 4.08 & 1.83 & $1.73,1.62$ & 3.25 & 7.21 & 4.38 & 58.76 & 28.85 & 27.30 & 43.42 \\
\hline & Gly & 8.71 & -5.8 & $4.11,3.72$ & & & & & & 46.86 & & & \\
\hline & Asp & 8.25 & -3.2 & 4.66 & 2.88 & & & & 8.41 & * & 38.80 & & \\
\hline & Aсрса 30 & \multicolumn{12}{|c|}{$7.60(\mathrm{NH}), 4.15(\mathrm{H} 3), 3.14(\mathrm{H} 1), 2.22,1.80(\mathrm{H} 5), 2.16,1.81(\mathrm{H} 2), 1.77(\mathrm{H} 4), \Delta \delta / \Delta \mathrm{T}=-3.6,{ }^{3} J_{\mathrm{NH}-\alpha \mathrm{H}}=6.1$} \\
\hline & & \multicolumn{12}{|c|}{55.25 (C3), $44.26(\mathrm{C} 1), 35.48(\mathrm{C} 2), 35.03(\mathrm{C} 4), 30.54$ (C5) } \\
\hline \multirow[t]{5}{*}{ Compd 9} & Arg & 8.40 & -5.6 & 4.00 & 1.79 & $1.68,1.60$ & 3.22 & 7.16 & 5.40 & 57.73 & 28.91 & 27.27 & 43.44 \\
\hline & Gly & 8.63 & -4.8 & $4.17,3.54$ & & & & & & 46.70 & & & \\
\hline & Asp & 8.17 & -2.4 & 4.64 & 2.83 & & & & 8.50 & * & 37.64 & & \\
\hline & Acpca 31 & \multicolumn{12}{|c|}{$7.35(\mathrm{NH}), 4.22(\mathrm{H} 3), 3.01(\mathrm{H} 1), 2.04(\mathrm{H} 2), 2.00,1.84(\mathrm{H} 5), 2.00,1.73(\mathrm{H} 4),-\Delta \delta / \Delta \mathrm{T}=-3.3,{ }^{3} J_{\mathrm{NH}-\alpha \mathrm{H}}=7.53$} \\
\hline & & \multicolumn{12}{|c|}{$54.33(\mathrm{C} 3), 45.02(\mathrm{C} 1), 39.85(\mathrm{C} 2), 34.76(\mathrm{C} 4), 29.03(\mathrm{C} 5)$} \\
\hline \multirow[t]{5}{*}{ Compd 10} & Arg & 8.42 & -7.16 & 4.48 & $1.89,1.77$ & $1.66,1.62$ & 3.22 & 7.23 & 7.20 & 55.79 & 31.77 & 27.37 & 43.38 \\
\hline & Gly & 8.61 & -5.46 & $4.01,3.84$ & & & & & & 45.62 & & & \\
\hline & Asp & 8.42 & -3.74 & 4.60 & $2.91,2.82$ & & & & 6.80 & * & 37.76 & & \\
\hline & Acpca 32 & \multicolumn{12}{|c|}{$7.57(\mathrm{NH}), 4.22(\mathrm{H} 3), 2.90(\mathrm{H} 1), 2.05,1.65(\mathrm{H} 4), 1.94,1.88(\mathrm{H} 2), 1.93,1.78(\mathrm{H} 5),-\Delta \delta / \Delta \mathrm{T}=-2.88,{ }^{3} J_{\mathrm{NH}-\alpha \mathrm{H}}=6.80$} \\
\hline & & \multicolumn{12}{|c|}{$51.00(\mathrm{C} 3), 45.48(\mathrm{C} 1), 40.06(\mathrm{C} 2), 34.00(\mathrm{C} 4), 30.11(\mathrm{C} 5)$} \\
\hline \multirow[t]{5}{*}{ Compd 11} & Arg & 8.27 & -7.6 & 4.39 & $1.87,1.77$ & $1.69,1.64$ & 3.23 & 7.21 & 7.33 & 55.79 & 31.28 & 27.35 & 43.46 \\
\hline & Gly & 8.71 & -6.8 & $3.94,3.80$ & & & & & & 46.31 & & & \\
\hline & Asp & 8.47 & -6.1 & 4.57 & 2.93 & & & & 7.45 & 53.17 & 38.06 & & \\
\hline & Acpca 33 & \multicolumn{12}{|c|}{$7.47(\mathrm{NH}), 4.22(\mathrm{H} 3), 3.00(\mathrm{H} 1), 2.10,1.68(\mathrm{H} 5), 2.05,1.57(\mathrm{H} 4), 2.03,1.70(\mathrm{H} 2), \Delta \delta / \Delta \mathrm{T}=-1.0,{ }^{3} J_{\mathrm{NH}-\alpha \mathrm{H}}=7.53$} \\
\hline & & \multicolumn{12}{|c|}{$54.25(\mathrm{C} 3), 45.39(\mathrm{Cl}), 37.14(\mathrm{C} 2), 35.29(\mathrm{C} 4), 31.97(\mathrm{C} 5)$} \\
\hline
\end{tabular}

* Resonances not observed due to water resonance overlap. 
Table S4. Torsion angles $\phi$ and $\psi$ (in degrees) of the leading members of the clusters (with more than 10 members) obtained from energy minimized conformers of $\mathbf{1 , 3}, \mathbf{6}, \mathbf{7}, \mathbf{9}$, and $\mathbf{1 0}$.

\begin{tabular}{|c|c|c|c|c|c|}
\hline Compd & & Angle & Arg & Gly & Asp \\
\hline 1 & Conf1 & $\phi, \psi$ & $-110,-65$ & $-90,-80$ & $-105,+60$ \\
\hline \multirow[t]{2}{*}{3} & Conf1 & $\phi, \psi$ & $-170,-65$ & $-95,-70$ & $-100,+70$ \\
\hline & Conf2 & $\phi, \psi$ & $-75,-60$ & $-95,-90$ & $-85,+80$ \\
\hline \multirow[t]{2}{*}{6} & Conf1 & $\phi, \psi$ & $+45,+65$ & $+135,-110$ & $-90,+60$ \\
\hline & Conf2 & $\phi, \psi$ & $-75,-60$ & $-100,-90$ & $-80,+85$ \\
\hline \multirow[t]{3}{*}{7} & Conf1 & $\phi, \psi$ & $+105,+80$ & $+115,-135$ & $-55,-55$ \\
\hline & Conf2 & $\phi, \psi$ & $-165,+45$ & $+90,+100$ & $+55,+75$ \\
\hline & Conf3 & $\phi, \psi$ & $-165,+60$ & $+110,-125$ & $-70,+105$ \\
\hline \multirow[t]{2}{*}{9} & Conf1 & $\phi, \psi$ & $-75,-55$ & $-105,-90$ & $-80,+85$ \\
\hline & Conf2 & $\phi, \psi$ & $+65,+75$ & $+120,-115$ & $-90,+65$ \\
\hline \multirow[t]{2}{*}{10} & Conf1 & $\phi, \psi$ & $-160,+65$ & $+90,+105$ & $+50,+60$ \\
\hline & Conf2 & $\phi, \psi$ & $+105,+90$ & $+115,-120$ & $-65,-75$ \\
\hline
\end{tabular}


${ }^{1} \mathrm{H}$ NMR spectrum $\left(600 \mathrm{MHz}, \mathrm{D}_{2} \mathrm{O}\right)$ of cyclopeptide $\mathbf{1}$
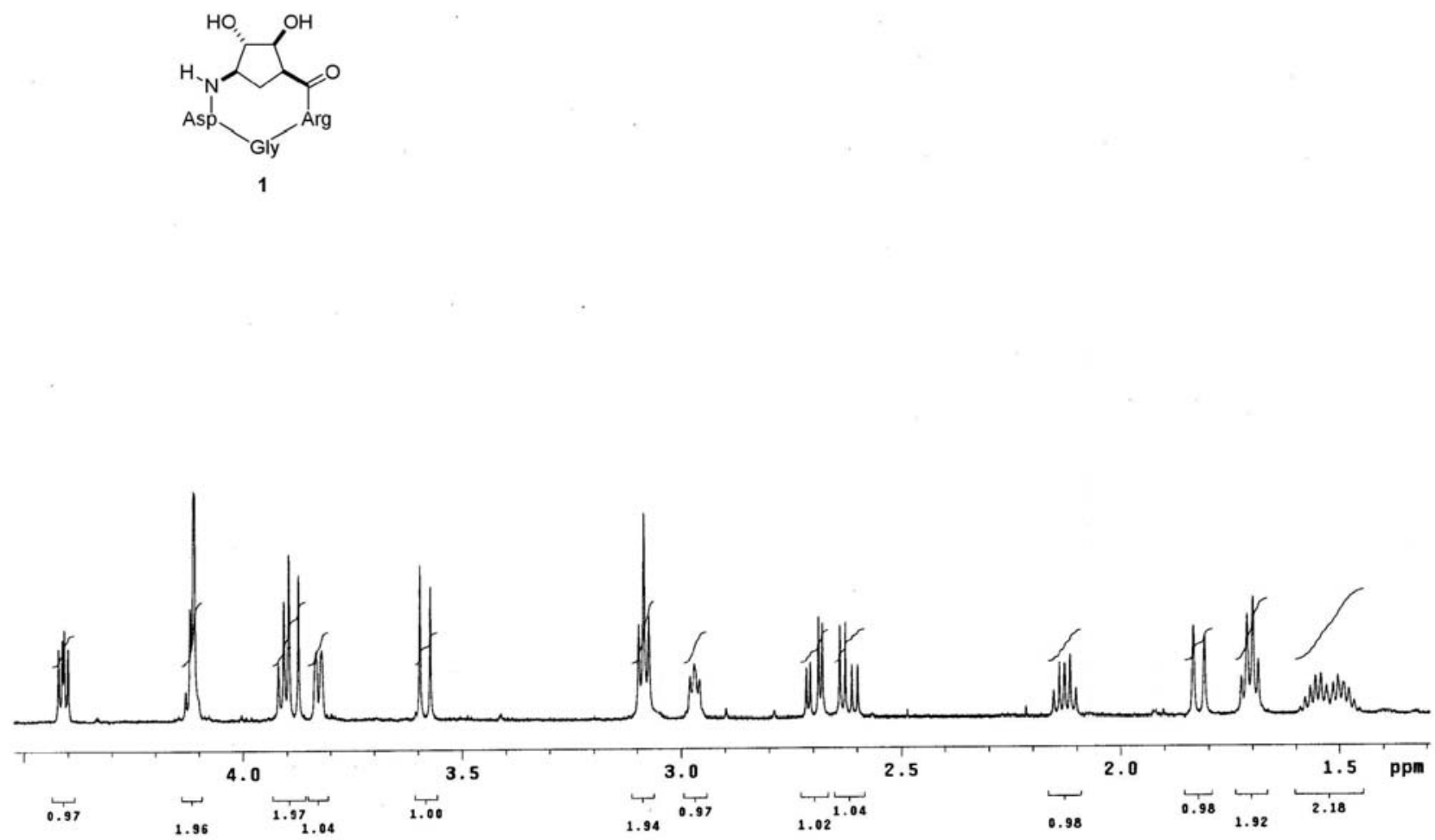
${ }^{1} \mathrm{H}$ NMR spectrum $\left(600 \mathrm{MHz}, \mathrm{D}_{2} \mathrm{O}\right)$ of cyclopeptide 2
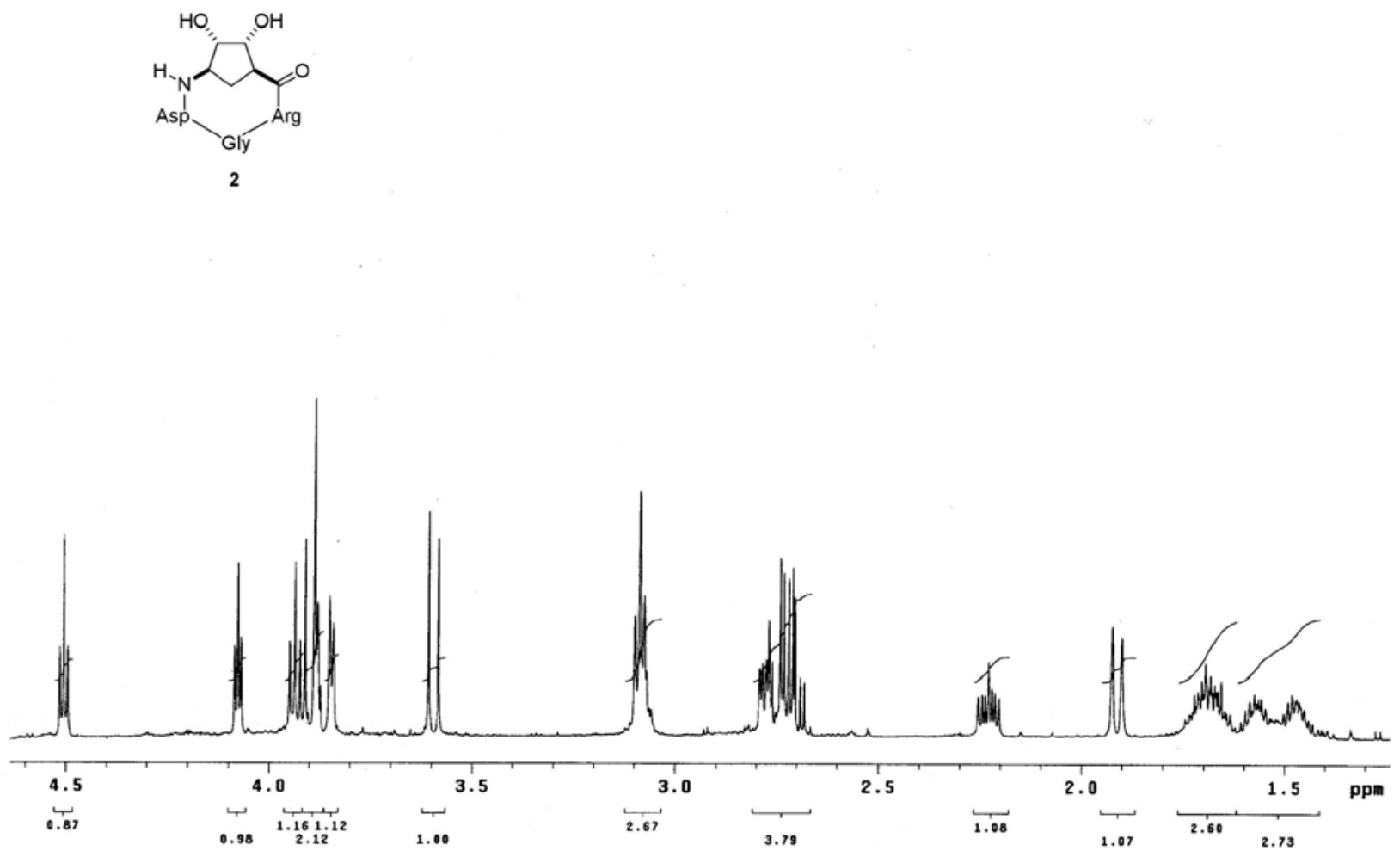
${ }^{1} \mathrm{H}$ NMR spectrum $\left(600 \mathrm{MHz}, \mathrm{D}_{2} \mathrm{O}\right)$ of cyclopeptide 3
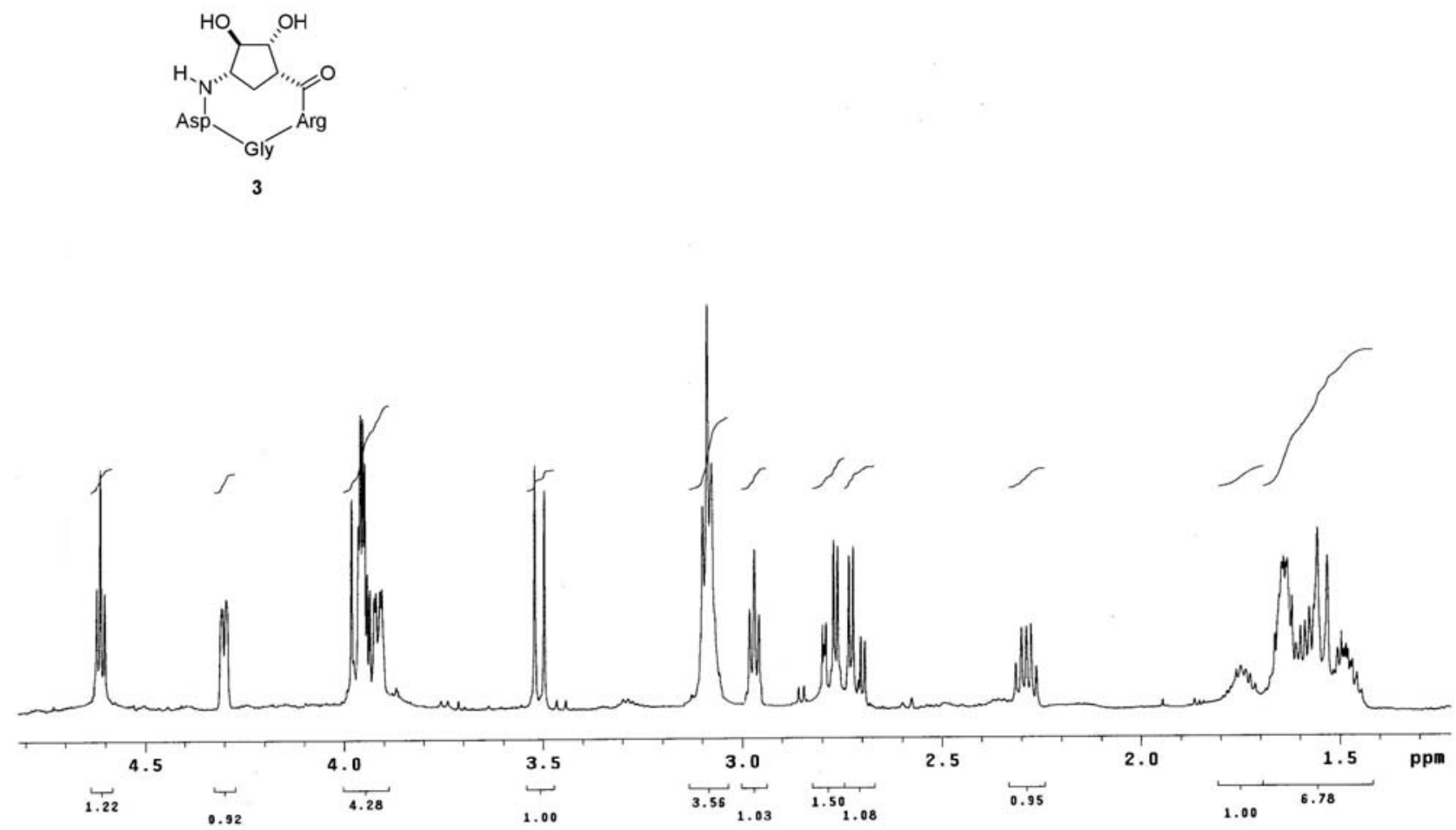
${ }^{1} \mathrm{H}$ NMR spectrum $\left(600 \mathrm{MHz}, \mathrm{D}_{2} \mathrm{O}\right)$ of cyclopeptide 4
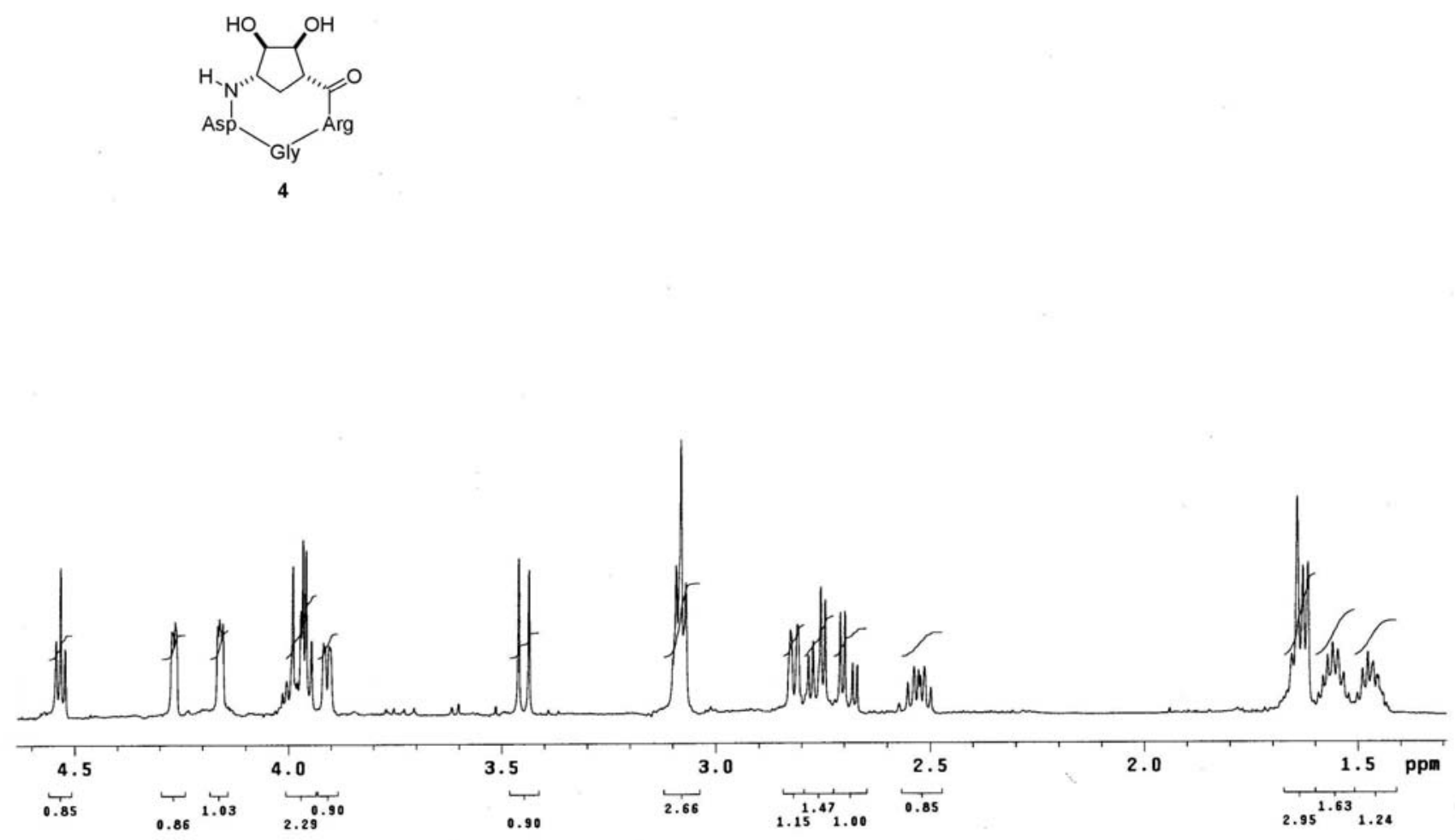
${ }^{1} \mathrm{H}$ NMR spectrum $\left(600 \mathrm{MHz}, \mathrm{D}_{2} \mathrm{O}\right)$ of cyclopeptide 5
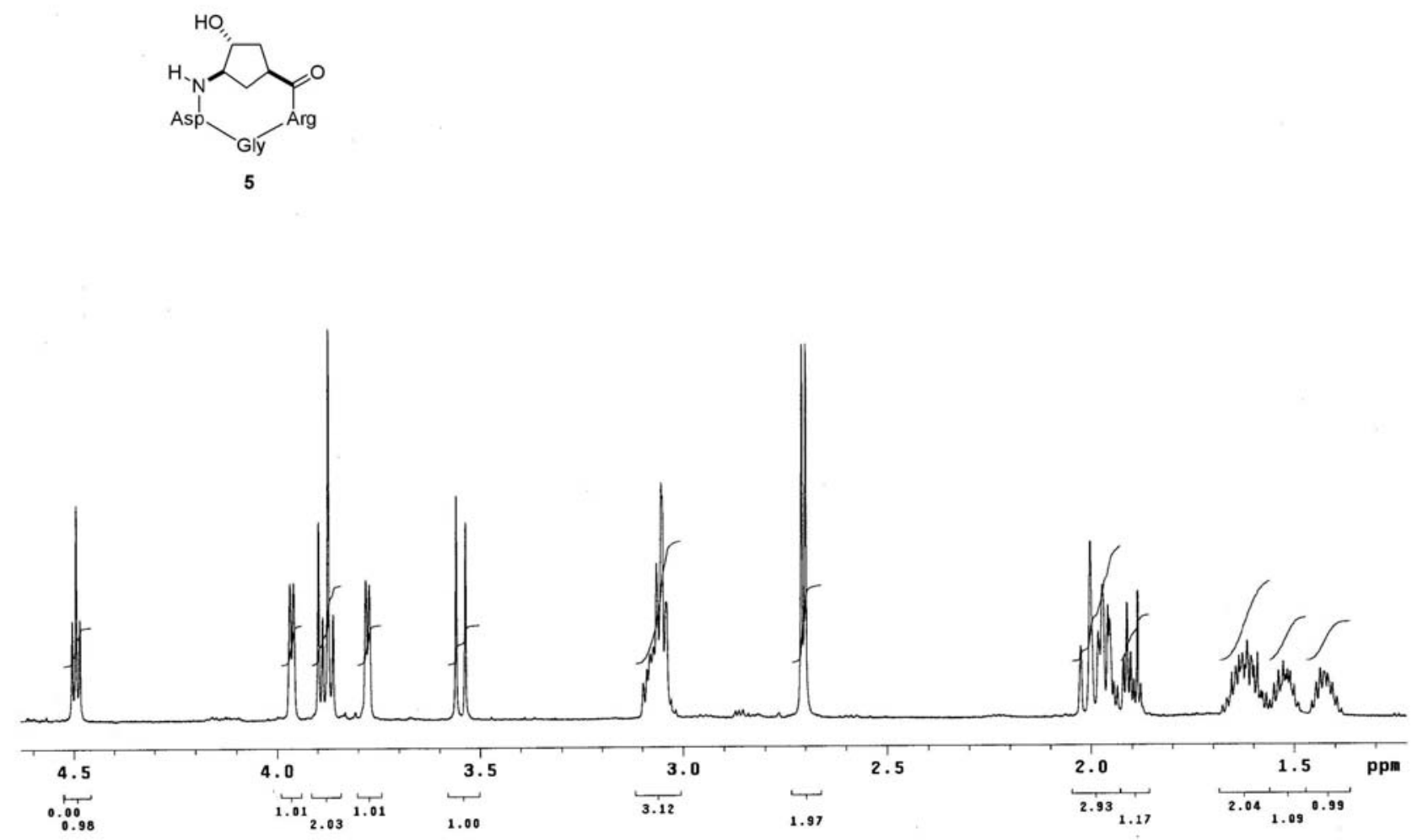
${ }^{1} \mathrm{H}$ NMR spectrum $\left(600 \mathrm{MHz}, \mathrm{D}_{2} \mathrm{O}\right)$ of cyclopeptide 6
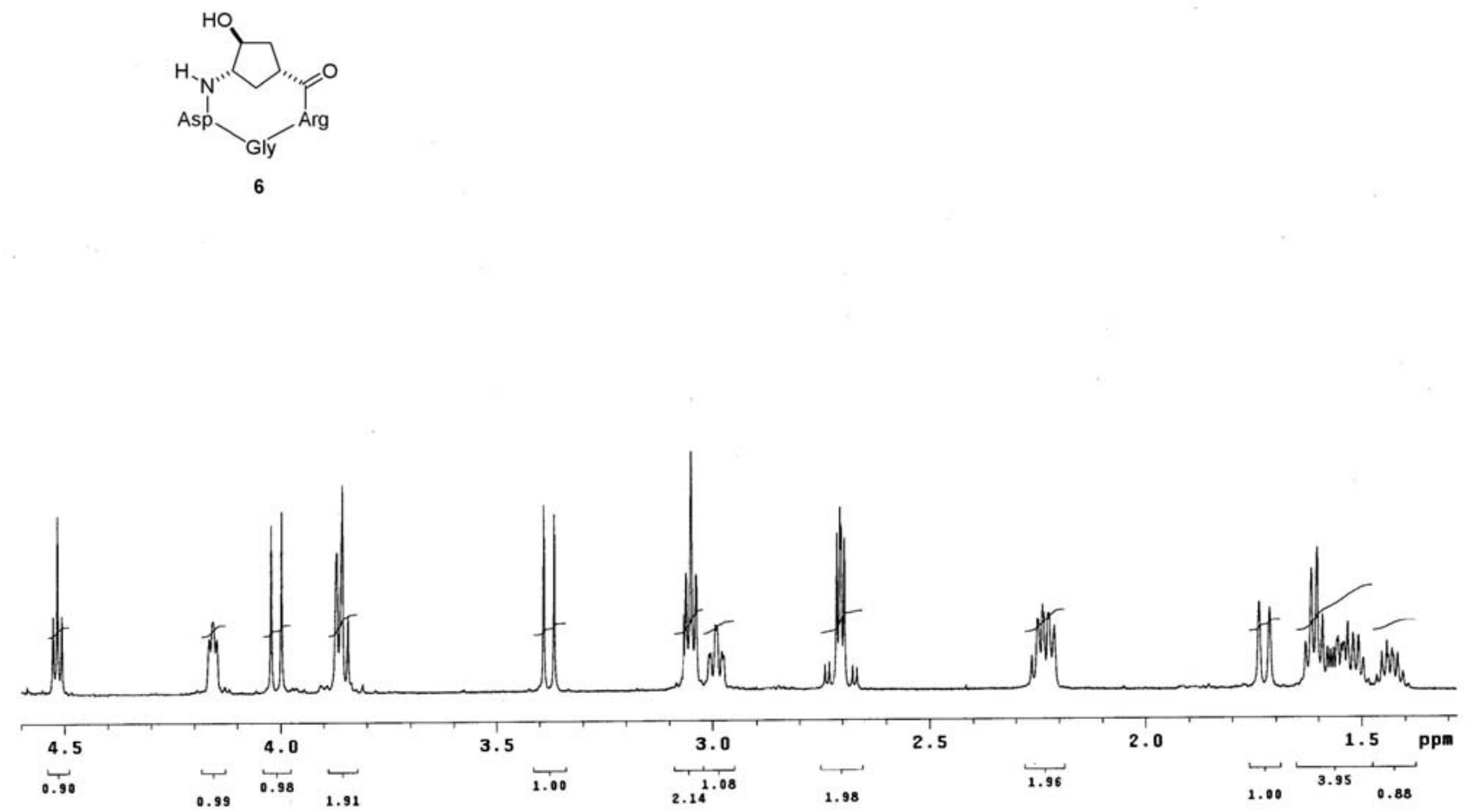
${ }^{1} \mathrm{H}$ NMR spectrum $\left(600 \mathrm{MHz}, \mathrm{D}_{2} \mathrm{O}\right)$ of cyclopeptide 7
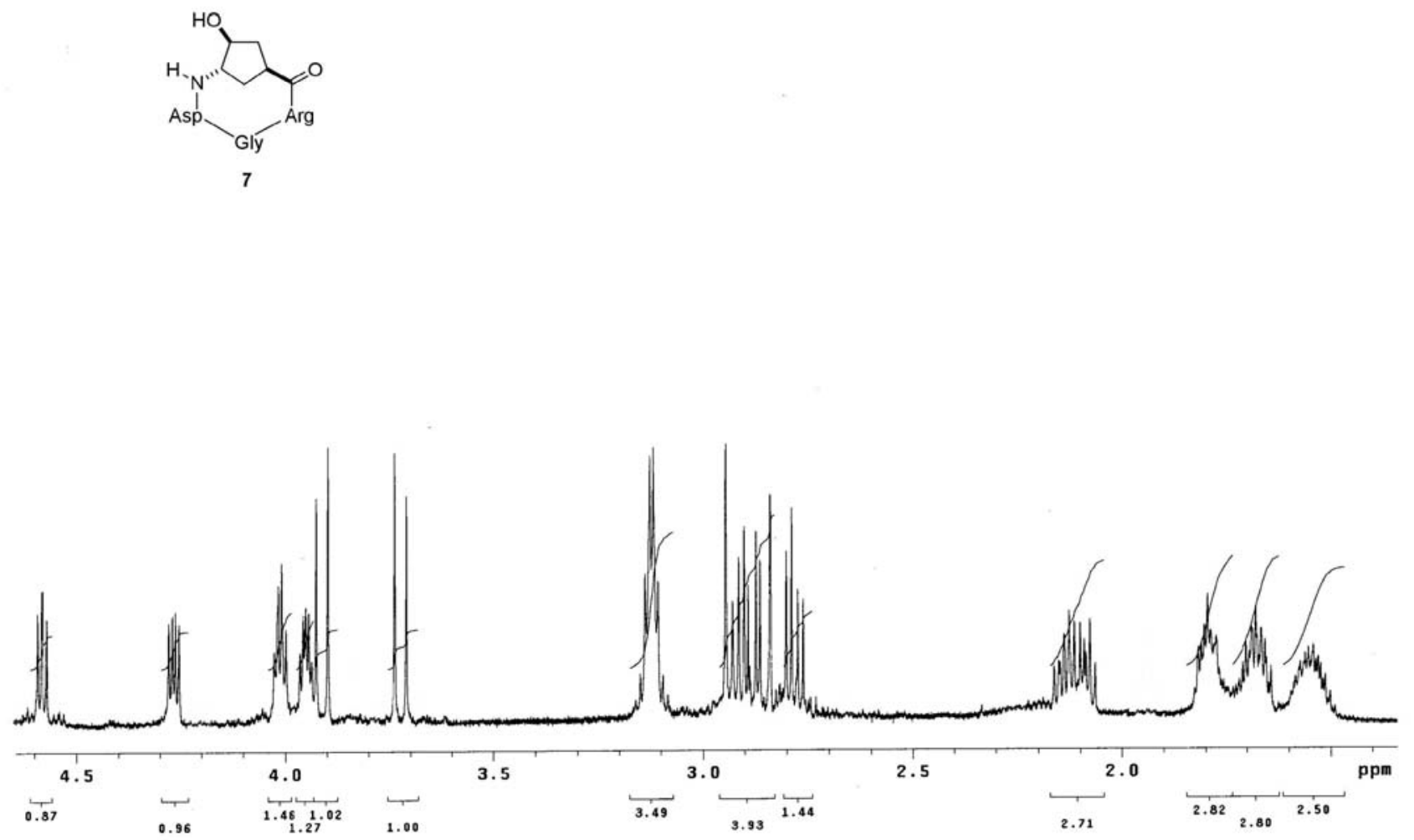
${ }^{1} \mathrm{H}$ NMR spectrum $\left(600 \mathrm{MHz}, \mathrm{D}_{2} \mathrm{O}\right)$ of cyclopeptide 8
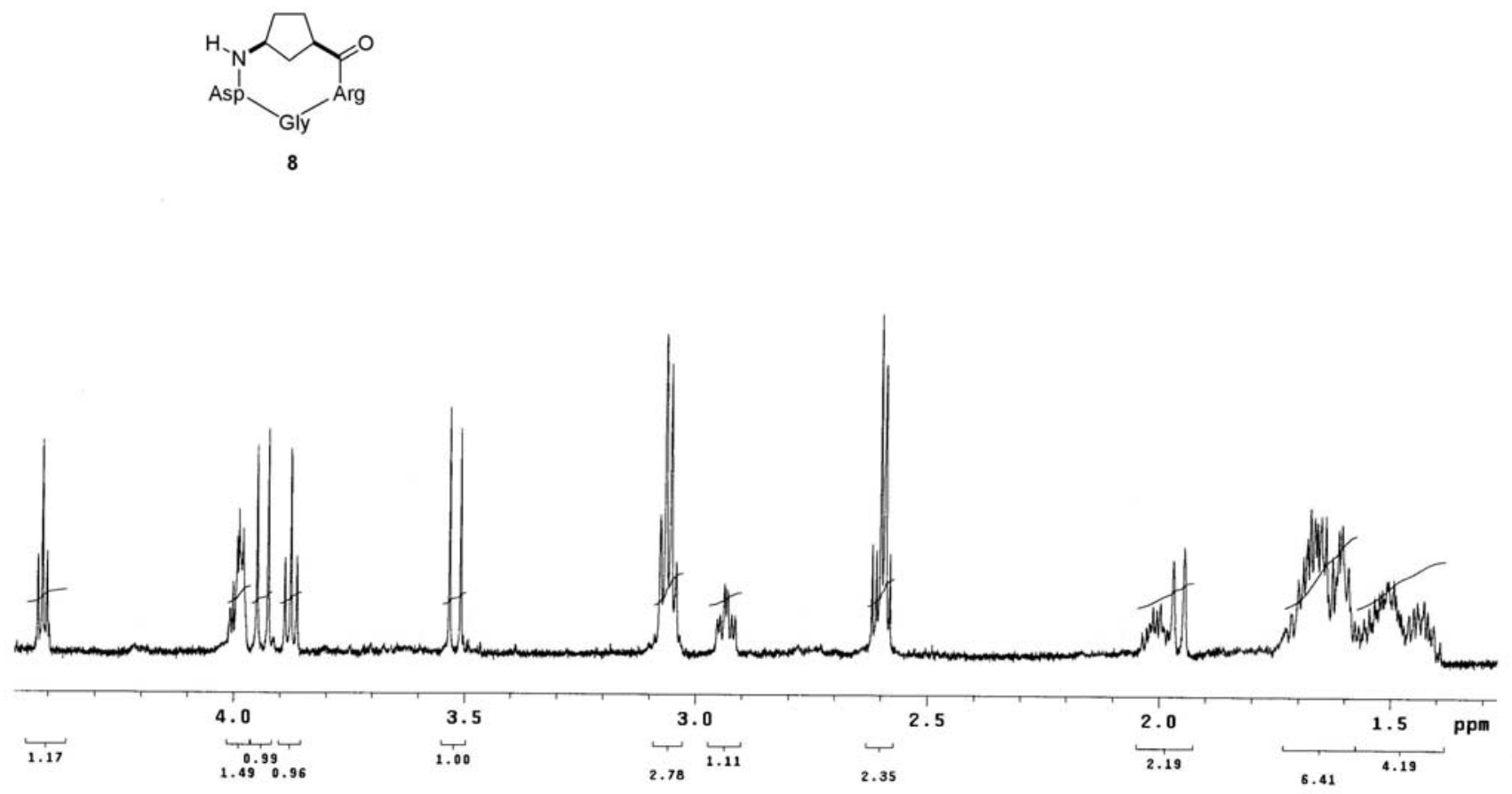
${ }^{1} \mathrm{H}$ NMR spectrum $\left(600 \mathrm{MHz}, \mathrm{D}_{2} \mathrm{O}\right)$ of cyclopeptide 9
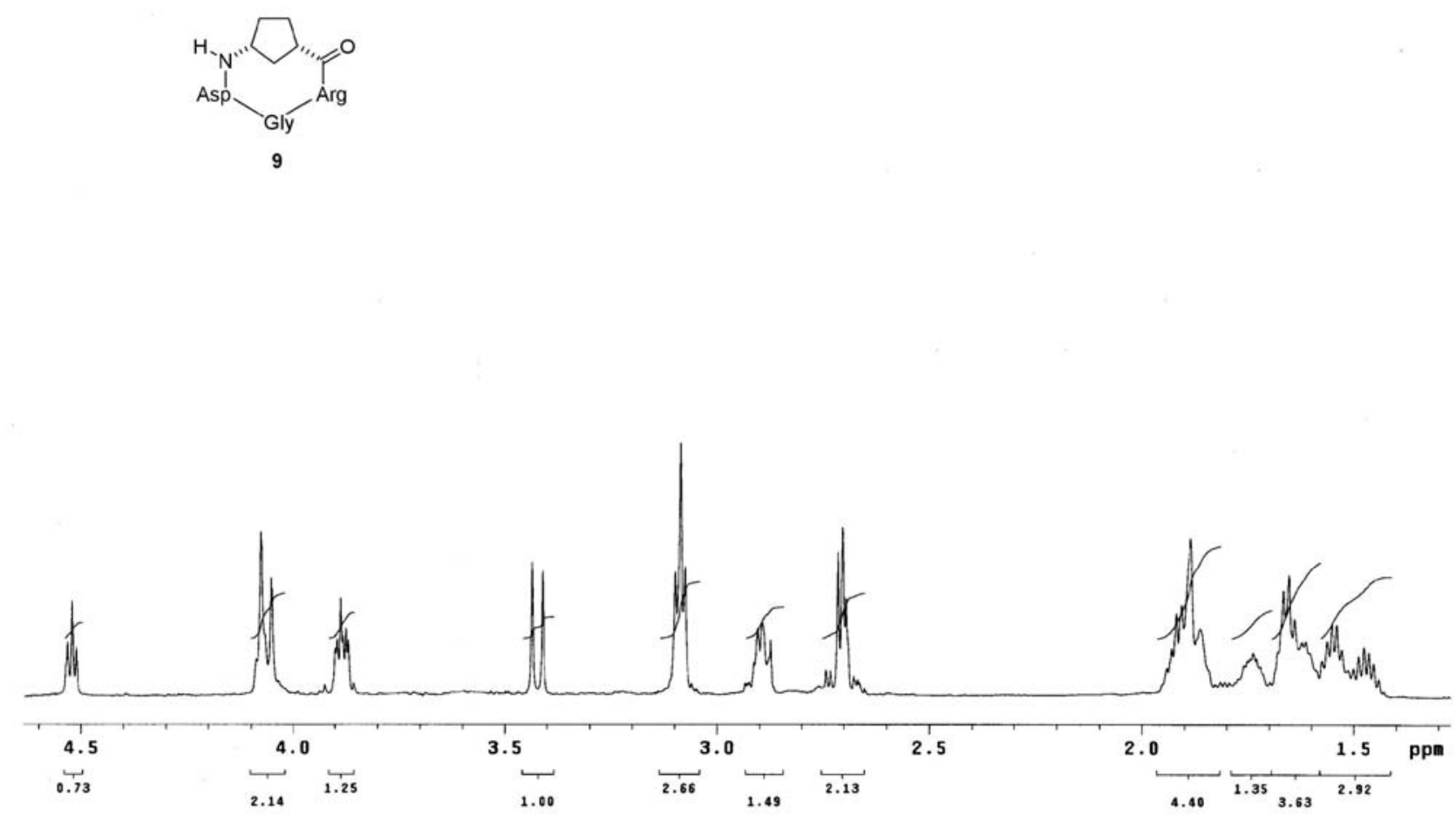
${ }^{1} \mathrm{H}$ NMR spectrum $\left(600 \mathrm{MHz}, \mathrm{D}_{2} \mathrm{O}\right)$ of cyclopeptide $\mathbf{1 0}$
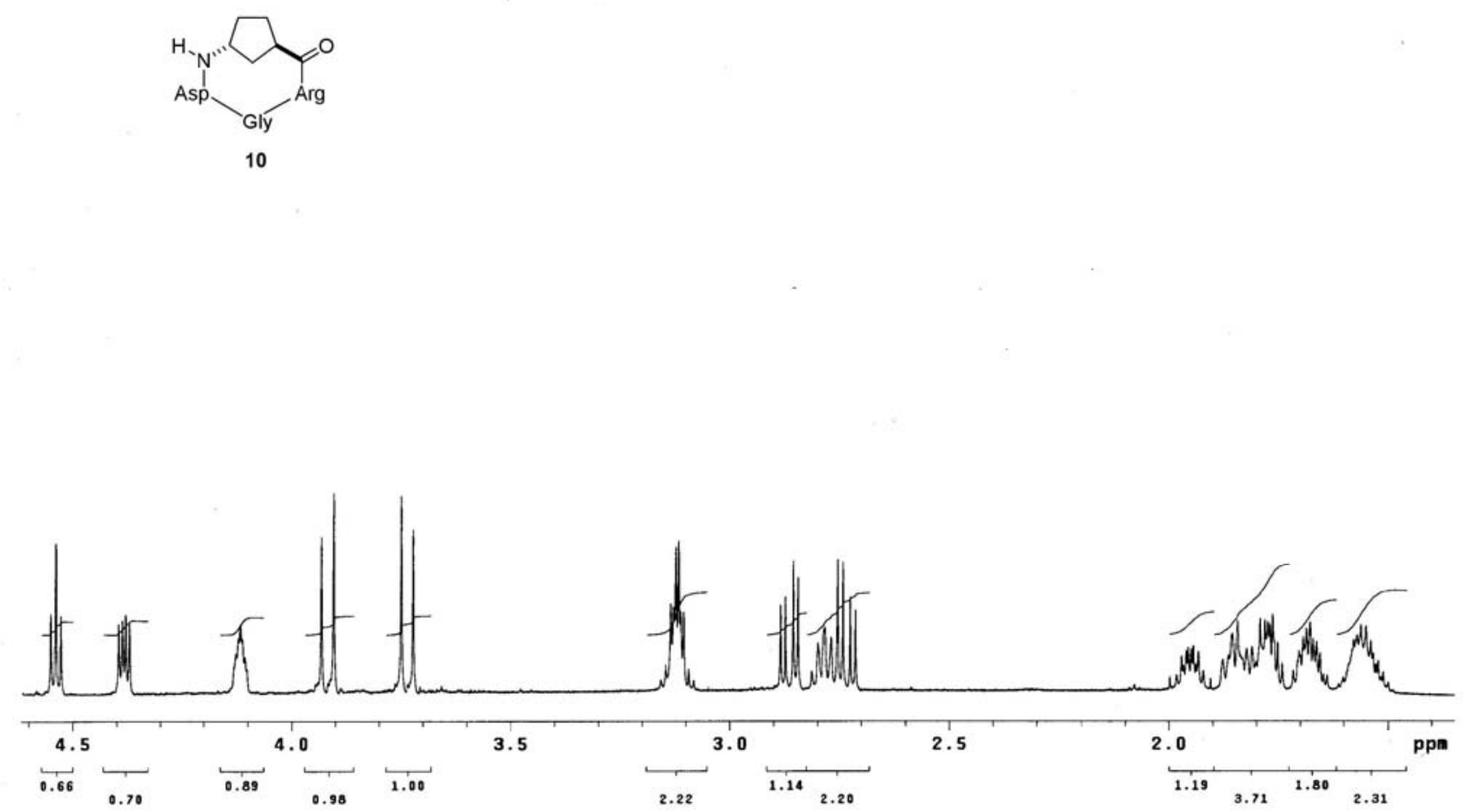
${ }^{1} \mathrm{H}$ NMR spectrum $\left(600 \mathrm{MHz}, \mathrm{D}_{2} \mathrm{O}\right)$ of cyclopeptide $\mathbf{1 1}$
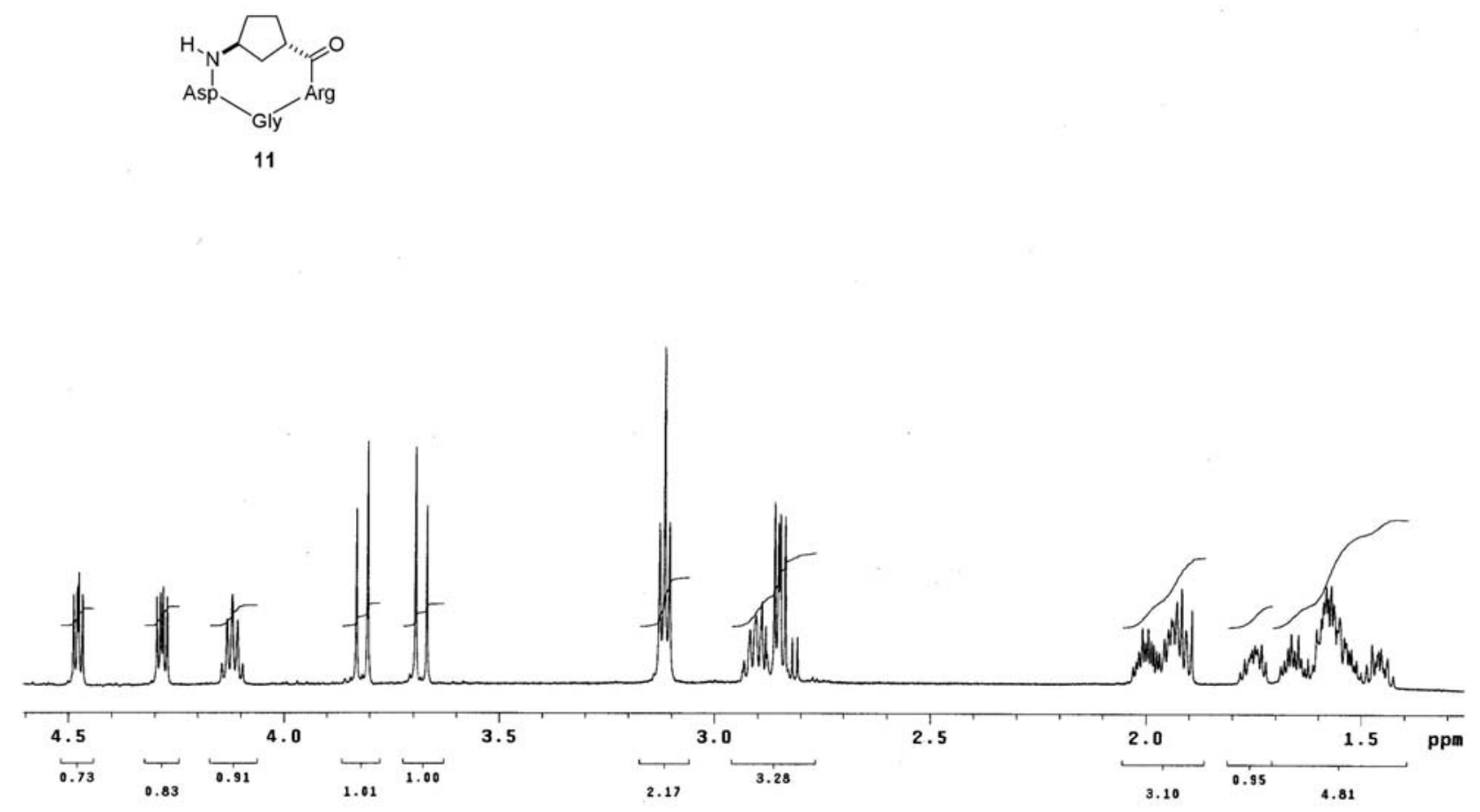
Table S5. Elemental analyses of relevant intermediary compounds.

\begin{tabular}{|c|c|c|c|c|c|c|c|}
\hline \multirow[b]{3}{*}{ Compound } & \multirow[b]{3}{*}{ Formula } & & & & & & \\
\hline & & \multicolumn{3}{|c|}{ Calcd (\%) } & \multicolumn{3}{|c|}{ Found (\%) } \\
\hline & & C & $\mathbf{H}$ & $\mathbf{N}$ & $\mathbf{C}$ & $\mathbf{H}$ & $\mathbf{N}$ \\
\hline 14 & $\mathrm{C}_{15} \mathrm{H}_{23} \mathrm{NO}_{6}$ & 57.50 & 7.40 & 4.47 & 57.31 & 7.35 & 4.32 \\
\hline 15 & $\mathrm{C}_{16} \mathrm{H}_{31} \mathrm{NO}_{4} \mathrm{Si}$ & 58.32 & 9.48 & 4.25 & 58.55 & 9.58 & 4.43 \\
\hline 16 & $\mathrm{C}_{19} \mathrm{H}_{29} \mathrm{NO}_{3} \mathrm{Si}$ & 65.67 & 8.41 & 4.03 & 65.58 & 8.55 & 4.11 \\
\hline 17 & $\mathrm{C}_{25} \mathrm{H}_{43} \mathrm{NO}_{3} \mathrm{Si}_{2}$ & 65.02 & 9.39 & 3.03 & 65.29 & 9.15 & 3.27 \\
\hline 18 & $\mathrm{C}_{25} \mathrm{H}_{43} \mathrm{NO}_{3} \mathrm{Si}_{2}$ & 65.02 & 9.39 & 3.03 & 65.26 & 9.61 & 3.22 \\
\hline 19 & $\mathrm{C}_{21} \mathrm{H}_{21} \mathrm{NO}_{6}$ & 65.79 & 5.52 & 3.65 & 65.52 & 5.77 & 3.44 \\
\hline 20 & $\mathrm{C}_{21} \mathrm{H}_{21} \mathrm{NO}_{6}$ & 65.79 & 5.52 & 3.65 & 65.95 & 5.74 & 3.41 \\
\hline 21 & $\mathrm{C}_{21} \mathrm{H}_{21} \mathrm{NO}_{6}$ & 65.79 & 5.52 & 3.65 & 65.86 & 5.69 & 3.58 \\
\hline 22 & $\mathrm{C}_{21} \mathrm{H}_{21} \mathrm{NO}_{6}$ & 65.79 & 5.52 & 3.65 & 65.70 & 5.71 & 3.49 \\
\hline 30 & $\mathrm{C}_{21} \mathrm{H}_{21} \mathrm{NO}_{4}$ & 71.78 & 6.02 & 3.99 & 72.01 & 6.12 & 3.84 \\
\hline 31 & $\mathrm{C}_{21} \mathrm{H}_{21} \mathrm{NO}_{4}$ & 71.78 & 6.02 & 3.99 & 71.66 & 6.13 & 3.87 \\
\hline 32 & $\mathrm{C}_{21} \mathrm{H}_{21} \mathrm{NO}_{4}$ & 71.78 & 6.02 & 3.99 & 71.88 & 6.15 & 4.12 \\
\hline 33 & $\mathrm{C}_{21} \mathrm{H}_{21} \mathrm{NO}_{4}$ & 71.78 & 6.02 & 3.99 & 71.91 & 5.96 & 4.07 \\
\hline 34 & $\mathrm{C}_{21} \mathrm{H}_{21} \mathrm{NO}_{5}$ & 68.65 & 5.76 & 3.81 & 68.73 & 5.91 & 3.69 \\
\hline 35 & $\mathrm{C}_{21} \mathrm{H}_{21} \mathrm{NO}_{5}$ & 68.65 & 5.76 & 3.81 & 68.52 & 5.93 & 3.75 \\
\hline 36 & $\mathrm{C}_{21} \mathrm{H}_{21} \mathrm{NO}_{5}$ & 68.65 & 5.76 & 3.81 & 68.78 & 5.81 & 3.63 \\
\hline
\end{tabular}




\section{References}

(1) Rassu, G.; Zanardi, F.; Battistini, L.; Gaetani, E.; Casiraghi, G. Expeditious syntheses of sugarmodified nucleosides and collections thereof exploiting furan-, pyrrole-, and thiophene-based siloxy dienes. J. Med. Chem. 1997, 40, 168-180.

(2) Zanardi, F.; Battistini, L.; Rassu, G.; Auzzas, L.; Pinna, L.; Marzocchi, L.; Acquotti, D.; Casiraghi, G. The Utility of Furan, Pyrrole, and Thiophene 2-Silyloxy Dienes As Demonstrated by Modular Synthesis of Annonaceous Acetogenin Core Units and Their Pyrrolidine and Thiolane Analogues. J. Org. Chem. 2000, 65, 2048-2064.

(3) Hubschwerlen, C.; Specklin, J:-L.; Higelin, J. L-(S)-Glyceraldehyde Acetonide. Org. Synth. 1995, 72, 1-5. 